STRUCTURAL SCIENCE CRYSTAL ENGINEERING MATERIALS

ISSN 2052-5206

Received 10 January 2019

Accepted 29 June 2019

Edited by J. Lipkowski, Cardinal Stefan Wyszynski University, Poland

Keywords: nanowires; zincblende structure; diamond structure; analytical descriptions.

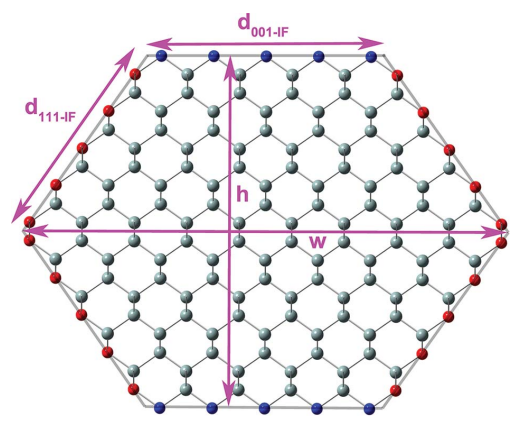

C 2019 International Union of Crystallography

\section{Analytical description of nanowires. I. Regular cross sections for zincblende and diamond structures}

\author{
Dirk König $^{\mathrm{a}, \mathrm{b} *}$ and Sean C. Smith \\ antegrated Materials Design Centre (IMDC), UNSW Node, University of New South Wales, NSW 2052, Australia, \\ ${ }^{\mathbf{b}}$ Institute of Semiconductor Electronics (IHT), RWTH Aachen University, 52074 Aachen, Germany, and ${ }^{\mathbf{c}}$ Department of \\ Applied Mathematics, Research School of Physics and Engineering, Australian National University, ACT 2601, Australia. \\ *Correspondence e-mail: solidstatedirk@gmail.com
}

Semiconductor nanowires (NWires) experience stress and charge transfer from their environment and impurity atoms. In response, the environment of a NWire experiences a NWire stress response which may lead to propagated strain and a change in the shape and size of the NWire cross section. Here, geometric number series are deduced for zincblende- (zb-) and diamond-structured NWires of diameter $d_{\text {Wire }}$ to obtain the numbers of NWire atoms $N_{\text {Wire }}\left(d_{\text {Wire }}[i]\right)$, bonds between NWire atoms $N_{\text {bnd }}\left(d_{\text {Wire }}[i]\right)$ and interface bonds $N_{\mathrm{IF}}\left(d_{\text {Wire }}[i]\right)$ for six high-symmetry zb NWires with the low-index faceting that occurs frequently in both bottom-up and top-down approaches of NWire processing. Along with these primary parameters, the specific lengths of interface facets, the crosssectional widths and heights and the cross-sectional areas are presented. The fundamental insights into NWire structures revealed here offer a universal gauge and thus could enable major advancements in data interpretation and understanding of all zb- and diamond-structure-based NWires. This statement is underpinned with results from the literature on cross-section images from III-V core-shell NWire growth and on Si NWires undergoing self-limiting oxidation and etching. The massive breakdown of impurity doping due to self-purification is shown to occur for both Si NWires and Si nanocrystals (NCs) for a ratio of $N_{\text {bnd }} / N_{\text {Wire }}=N_{\text {bnd }} / N_{\text {NC }}=1.94 \pm 0.01$ using published experimental data.

\section{Introduction}

It is well known that the electronic structure and optical response of nanoscale systems such as nanowires (NWires) or nanocrystals (NCs) is a function of lattice strain which is routinely measured by Raman and Fourier transform infra-red (FT-IR) spectroscopy to probe phononic spectra (Jaccodine \& Schlegel, 1966; Anastassakis et al., 1970; Nakashima et al., 1981; Boyd \& Wilson, 1982, 1987). Such spectra are sensitive to lattice strain which is a function of the material via the Young's modulus (Elliot, 1998). Changes in compressive or expansive stress were shown to modify the optical response of NWires by photoluminescence (PL) (Tomioka et al., 2011; Joyce et al., 2011; Treu et al., 2015). The growth of monolithic NWires depends critically on balanced stress to avoid stacking faults which cause the electronic properties of the NWire to deteriorate. Attempts to place phosphorus atoms as donors on lattice sites in free-standing $\mathrm{Si}$ NCs were shown to fail increasingly with shrinking NC diameter (Stegner et al., 2008, 2009; König et al., 2015; Gnaser et al., 2014). As a structural cause, self-purification was identified (Dalpian \& Chelikowsky, 2006, 2008; Chan et al., 2008; Ossicini et al., 2005). While no detailed analogue investigations have yet been carried out on Si NWires, it is apparent from the selfpurification mechanism that a structural limit also exists for $\mathrm{Si}$ 


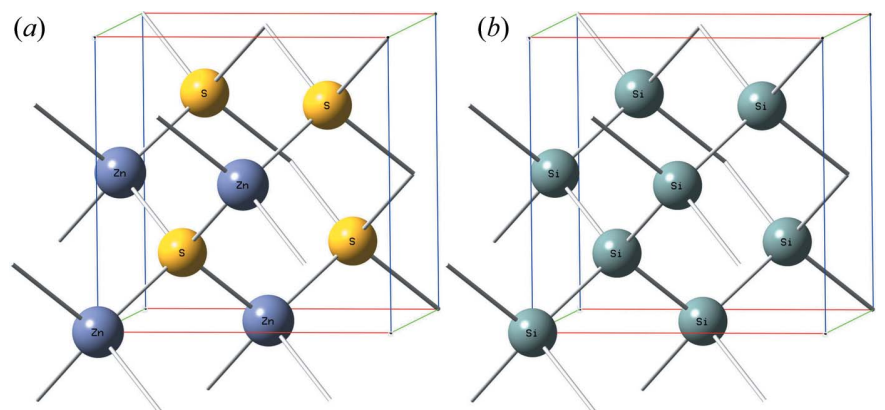

Figure 1

Examples of the periodic unit cells (UCs) of space groups $F \overline{4} 3 m(\mathrm{zb})$ and $F d \overline{3} m$ (diamond) covered in this work. (a) Zinc sulfide (Zinkblende) ZnS, standing for many II-VI and III-V semiconductors obeying the zb structure symmetry. (b) Main-group IV semiconductors such as diamond, germanium or silicon obey the diamond structure symmetry. Both space groups share the same crystal symmetry apart from their primitive basis, which is $A-A(\mathrm{Si}-\mathrm{Si})$ for diamond structures and $A-B(\mathrm{Zn}-\mathrm{S})$ for $\mathrm{zb}$ structures.

NWires and, to a lesser extent, for III-V NWires. All these structures are key to future electronic devices such as radial light-emitting diodes (Tomioka et al., 2011) or next-generation very-large-scale integration (VLSI) Si field-effect transistors (FETs) (Weber \& Mikolajick, 2017). An analytical structural description of the NWire cross section down to the individual bonds and atoms is a very powerful tool to interpret any experimental data as a function of the NWire's cross-section size and shape and the orientation of its growth axis and interfaces.

The goal of this work is to provide such an analytical description of cross sections for NWires consisting of zincblende- (zb-) and diamond-structured materials (Fig. 1) down to the individual atom/bond. These number series can deliver crucial input to interpret experimental and theoretical data from such NWire systems. A high accuracy in predicting NWire cross sections requires adequate experimental data such as exact lattice positions or interface-specific defect ratios. Alternatively, iterative techniques can be used (König et al., 2007).

With a universal gauge for stress, such phenomena could be scaled as a function of NWire diameter $d_{\text {Wire }}$. Principal parameters are the number of atoms forming the zb-NWire $N_{\text {Wire }}$, the bonds between such atoms $N_{\text {bnd }}$ and the number of bonds $N_{\text {IF }}$ terminating the NWire interface. This last can be broken down into the number of interface bonds per facet orientation $\{a b c\}$, viz. $N_{\mathrm{IF}}^{a b c}$. As parameters for size and shape identification, the characteristic interface lengths $d_{a b c \text {-IF }}$ per interface orientation $\{a b c\}$ serve as an identification tool for assigning experimental data to the respective analytical NWire cross section. The total cross-section area $A$ is a key parameter in particular for electronic devices where current densities can be used instead of absolute currents to interprete device behaviour. From these principal parameters, secondary parameters can be derived: the ratio $N_{\text {bnd }} / N_{\text {Wire }}$ yields the bonds per atom within a zb-NWire, the ratio $N_{\mathrm{IF}} / N_{\text {bnd }}$ yields the interface bonds per internal NWire bond, and the ratio $N_{\mathrm{IF}} /$ $N_{\text {Wire }}$ yields the interface bonds per NWire atom. For NWires with different interface faceting $\{a b c\}$ and $\{d e f\}$, ratios of interface bond densities per facet type $N_{\mathrm{IF}}^{a b c} / N_{\mathrm{IF}}^{\text {def }}$ can be derived.

Section 2 provides the necessary background information on the choice of NWire cross sections made, the interface bond densities and surface energies per major facet type, the nomenclature on how to interpret the cross-section images per NWire type, and a brief assignment of primary and secondary parameters to structurally driven phenomena. Section 3 contains the number series for all six different NWire cross sections. An associated Appendix $A$ shows the mathematical algorithm to derive these for the first NWire type which has an [001] growth axis, a square cross section and $\{001\}$ interfaces. In Section 4, we show an example of applying the number series and derived secondary parameters to experimental data from the literature for type III-V and Si NWires. Section 5 demonstrates the use of secondary parameters on more literature data over a broader base. Amongst these is a common material-specific limit of $N_{\text {bnd }} / N_{\text {Wire }}$ for both $\mathrm{Si}$ NWires and NCs, below which self-purification becomes dominant, and a lower size limit for NWire crystallization. Section 6 provides an outlook on follow-on analytical work, such as arbitrary cross-section morphing to fit experimental data exactly and the extension of the analytics to NWire materials with different space-group symmetries.

\section{General remarks on analytical number series, structural boundary conditions and nomenclature}

With $N_{\text {bnd }} / N_{\text {Wire }}$ we obtain a gauge for the response to internal stress, e.g. by dopant species. The ability of embedding materials or ligands to exert stress on NWires or vice versa can be described with $N_{\text {IF }} / N_{\text {bnd }}$. The impact of a highly polar surface termination on the zb-NWire electronic structure such as an interface charge transfer (König et al., 2014, 2018) is assessed by the ratio $N_{\mathrm{IF}} / N_{\text {Wire }}$. The ratio $N_{\mathrm{IF}}^{a b c} / N_{\mathrm{IF}}^{\text {def }}$ can be useful for detecting facet-specific interface defects.

We illustrate results from Si NWires (diamond structure). NWire atoms without interface bonds are shown in grey. Atoms with interface bonds are colour-coded: species with one, two or three interface bonds are red, blue or green, respectively; see Figs. 2 and 3 for examples. The analytical number series introduced below also hold for $\mathrm{zb}-\mathrm{NWires}$ due to straightforward symmetry arguments (Fig. 1). Hence, the analytical descriptions below cover all NWires with zb symmetry in addition to $\mathrm{Si}$ and Ge. Material properties resulting from differences in the base cell (see Fig. 1) are not considered here. This constraint has no impact on the applicability of the analytics of our work unless data of zb-type NWires are directly compared with diamond-type NWires.

Due to the complexity of symmetry arguments, zb-NWires with index faceting beyond $\{1 \overline{3} 1\}$ are not considered in our work. For Si, the $\{111\}(\{001\})$ facets have the lowest (secondlowest) experimental values of surface free energy (Table 1), followed by the $\{110\}$ facets. Regarding surface bond density, $\{111\}$-oriented facets have the lowest surface bond density for all facets up to $\{433\}$ orientation (Hesketh, 1993). These 
Table 1

Bond densities and free energies per square for low-index Si facets; bonddensity values taken from Hesketh (1993) and experimental surfaceenergy values taken from Eaglesham et al. (1993).

\begin{tabular}{lll}
\hline $\begin{array}{l}\text { Facet } \\
\text { orientation }\end{array}$ & $\begin{array}{l}\text { Surface bond density } \\
\left(\mathrm{cm}^{-2}\right)\end{array}$ & $\begin{array}{l}\text { Surface free energy } \\
\left(\mathrm{J} \mathrm{m}^{-2}\right)\end{array}$ \\
\hline$\{001\}$ & $1.36 \times 10^{15}$ & 1.36 \\
$\{110\}$ & $0.96 \times 10^{15}$ & 1.43 \\
$\{111\}$ & $0.78 \times 10^{15}$ & 1.23 \\
\hline
\end{tabular}

Table 2

The slab thickness $d_{\text {slab }}$ of NWire cross sections as a function of growthaxis orientation given in UC lengths $a_{\mathrm{UC}}$ per growth orientation to achieve periodicity; numbers of atoms and of bonds per column as described per feature seen in cross-section top view are given to enable the counting of atoms and NWire internal bonds.

\begin{tabular}{llll}
\hline $\begin{array}{l}\text { Growth } \\
\text { axis }\end{array}$ & $\begin{array}{l}d_{\text {slab }} \\
\left(a_{\mathrm{UC}}\right)\end{array}$ & $\begin{array}{l}\text { Atoms per } \\
\text { column in } \\
\text { top view }\end{array}$ & $\begin{array}{l}\text { Bonds per } \\
\text { column in } \\
\text { top view }\end{array}$ \\
\hline 001 & 1 & 1 & 1 per $\backslash$ and $/$ \\
110 & $\sqrt{2}$ & 2 & 2 per $/$ and $\backslash, 4$ per $\mid$ \\
111 & $\sqrt{3}$ & 2 & $\begin{array}{l}\text { per atom column, } 1 \\
\text { between atom columns }\end{array}$ \\
$11 \overline{2}$ & $\sqrt{6}$ & 1 & 2 per $X, 1$ per - and $-\dagger$ \\
\hline
\end{tabular}

$\dagger$ Bond symbols must be turned by $90^{\circ}$ to align with the graphs in Figs. 6 and 7 .

findings also hold for other diamond- and zb-NWires due to symmetry arguments.

The number series follow a run index $i$ which relates to $d_{\text {Wire }}[i]$ via the respective NWire cross-section area $A[i]$. Since the NWires considered here differ in their cross-section shape, we use $d_{\text {Wire }}$ of a circular NWire which allows a comparison of the same parameter of different NWire shapes as a function of $d_{\text {Wire }}^{\text {circ }}$,

$$
d_{\text {Wire }}^{\text {circ }}[i]=\sqrt{\frac{4}{\pi} A[i]} .
$$

This generalized size parameter $d_{\text {Wire }}^{\text {circ }}[i]$ becomes relevant in Section 5.

Below, we provide a general procedure to pick the correct NWire cross section to use for interpreting experimental data as a function of NWire structure. For direct imaging, e.g. by high-resolution transmission electron microscopy (HR-TEM, see Fig. 13) or scanning TEM (STEM, see Fig. 12), the ratio of interface lengths $d_{\mathrm{IF}}$, widths $w$ and heights $h$ of NWire cross sections can be readily extracted. If atomic information is available, as is the case in Fig. 13, it helps to split each NWire cross section further to arrive at two different symmetry centres, which is reflected in an even and an odd set of number series. Having two different symmetry centres available is useful for ultra-small NWire cross sections where such differences become notable. At such NWire diameters, the surface energies and associated wider angles (less-vulnerable edges) between the surface planes suggest that the cross sections are mainly hexagonal, which appears to be supported by experimental findings. In contrast, rectangular or square cross sections do not appear to have been found for NWire diameters at the lower end of the nanometre scale. While a
Table 3

The parameter list for each NWire cross section; all $N_{v}$ are calculated as per NWire slab.

\begin{tabular}{ll}
\hline Parameter & Description \\
\hline$N_{\text {Wire }}$ & No. of atoms forming the NWire \\
$N_{\text {bnd }}$ & No. of bonds within the NWire \\
$N_{\mathrm{IF}}$ & No. of interface bonds of the NWire \\
$N_{a b c-\mathrm{IF}}$ & No. of bonds per IF type $\{a b c\}$ \\
$d_{a b c-\mathrm{IF}}$ & Length of IF with orientation $\{a b c\}$ \\
$w$ & Maximum width of the NWire cross section \\
$h$ & Maximum height of the NWire cross section \\
$A$ & Cross-section area \\
\hline
\end{tabular}

Table 4

A list of the NWire shape indices [cross section, growth direction and side interfaces (where necessary)] added to all parameters as a superscript.

\begin{tabular}{llll}
\hline Superscript & Growth axis & Cross section & Side interfaces $\dagger$ \\
\hline $001-\square$ & 001 & Square & \\
$110-\square$ & 110 & Rectangular & \\
$110-\square$ & 110 & Hexagonal & \\
$11 \overline{2}-\square$ & $11 \overline{2}$ & Hexagonal & \\
$111-\square \mid 110$ & 111 & Hexagonal & 111 \\
$111-\square \mid 11 \overline{2}$ & 111 & Hexagonal & $11 \overline{2}$ \\
\hline
\end{tabular}

$\dagger$ Only when required to distinguish cross sections.

group of even and odd series can also be derived for square and rectangular NWire cross sections, such cross sections are not likely to exist at the bottom end of the nanometre scale. Hence, we present just one group of number series for both cross-section types, which also serves to keep our work concise.

Other spectroscopic methods, such as electron paramagnetic resonance (EPR) or Raman spectroscopy, are sensitive to certain interface defects or modifications which either exist exclusively or with a very high probability at specific interface orientations. The partitions per interfacespecific defect can then be used to determine the ratios of side lengths to specify the NWire cross section. An example with EPR is given at the end of Section 6.

For the description of zb- and diamond-structured NWire cross sections, we consider slabs with a thickness $d_{\text {slab }}$ of one unit-cell (UC) thickness $a_{\mathrm{UC}}$ multiplied by the respective factor in the direction of NWire growth to achieve periodicity; values are summarized in Table 2 . In addition, Table 2 lists the number of atoms and bonds per column (i.e. per atom or bond visible) as a function of NWire axis orientation for a top view of the cross section, thereby allowing us to count atoms and bonds. Respective images are provided for all NWire crosssection types presented here. Table 3 shows all parameters described by number series for each cross-section type. The indexing of the NWire cross-section type is given as a superscript with its shape and growth direction (see Table 4).

For all equations in this section, the limits of the run index are $1 \leq i<\infty$ unless noted otherwise. We explain the mathematical tools used to derive the number series in Appendix $A$ with the NWire type in Section 3.1. Further details of the crystallographic algorithm can be obtained from our previous work (König, 2016). 


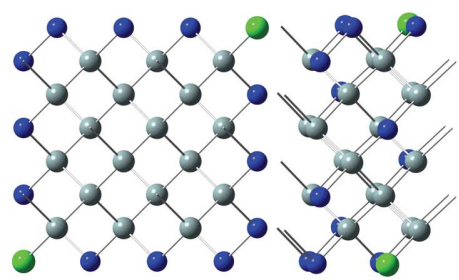

(a)

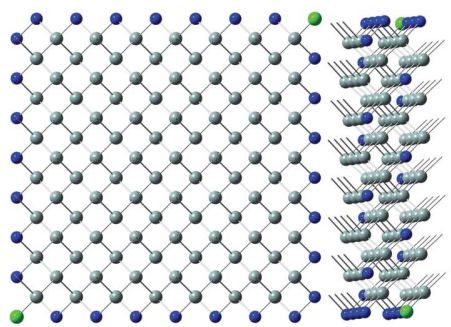

(c)

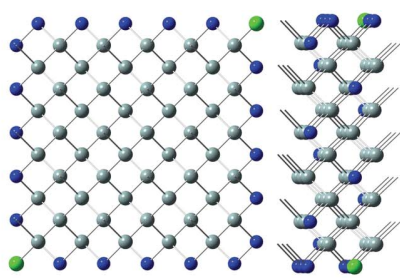

(b)
Figure 2

Cross sections and side views of $\mathrm{zb}$ - and diamond-structured NWires growing along the [001] axis with a square cross section and four $\{001\}$ interfaces for run indices $i=1$ to 4 . (a) $\mathrm{X}_{32},(b) \mathrm{X}_{72},(c) \mathrm{X}_{128}$ and $(d) \mathrm{X}_{200}$. Interior atoms are grey, atoms with two interface bonds are blue and atoms with three interface bonds are green.

\section{Analytical number series of nanowire cross sections}

3.1. NWires growing along the [001] direction with square cross section and four $\{001\}$ interfaces

$$
\begin{gathered}
N_{\mathrm{Wire}}^{001-\square}[i]=8(i+1)^{2}, \\
N_{\mathrm{bnd}}^{001-\square}[i]=(4 i+3)^{2}, \\
N_{\mathrm{IF}}^{001-\square}[i]=2(8 i+7), \\
d_{001-\mathrm{IF}}^{001-\square}[i]=a_{\mathrm{UC}}\left(i+\frac{3}{4}\right) .
\end{gathered}
$$

The square shape of the cross section results in $w[i] \equiv h[i] \equiv$ $d_{001-\mathrm{IF}}^{001-}[i]$.

$$
A^{001-\square}[i]=\left(a_{\mathrm{UC}}\right)^{2}\left(i+\frac{3}{4}\right)^{2} .
$$

Fig. 2 shows the first four members of the square NWire cross sections with growth along the [001] direction and four $\{001\}$ interfaces.

3.2. NWires growing along the [110] direction with rectangular cross section and two $\{001\}$ plus two $\{110\}$ interfaces

$$
\begin{aligned}
& N_{\text {Wire }}^{110-\square}[i]=8(i+1)\left(i+\frac{3}{2}\right), \\
& N_{\text {bnd }}^{110-\square}[i]=4(i+1)(4 i+3),
\end{aligned}
$$

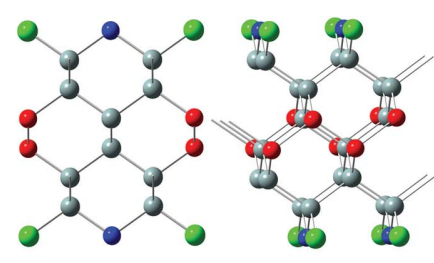

(a)

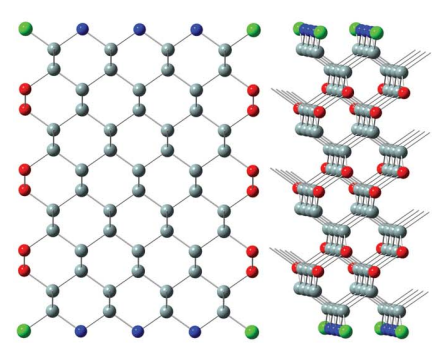

(c)

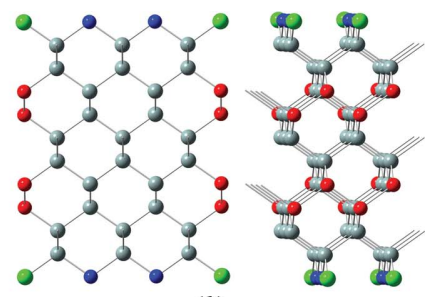

(b)

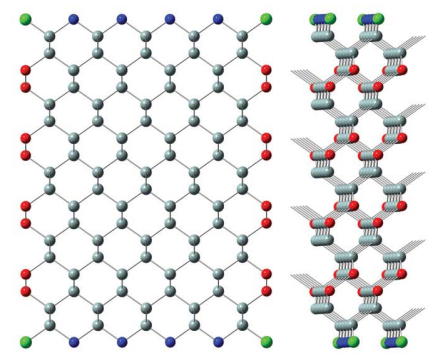

(d)
Figure 3

Cross sections and side views of zb- and diamond-structured NWires growing along the [110] axis with a rectangular cross section and two $\{111\}$ and two $\{001\}$ interfaces for run indices $i=1$ to 4 . (a) $\mathrm{X}_{40},(b) \mathrm{X}_{84},(c) \mathrm{X}_{144}$ and $(d) \mathrm{X}_{220}$. Interior atoms are grey, and atoms with one, two or three interface bonds are red, blue or green, respectively.

$$
\begin{gathered}
N_{\mathrm{IF}}^{110-\square}[i]=8(2 i+3), \\
\frac{N_{110-\mathrm{IF}}^{110-\square}[i]}{N_{001-\mathrm{IF}}^{110-\square}[i]}=\frac{8 i}{8(i+3)}=\frac{i}{i+3} .
\end{gathered}
$$

The central expression of equation (10) shows both number series in their explicit form, while the right-hand expression presents the simplified result of their ratio.

$$
d_{001-\mathrm{IF}}^{110-\square}[i]=a_{\mathrm{UC}}\left(\frac{i+1}{\sqrt{2}}\right) .
$$

The width of the rectangular cross section follows straightforwardly from $w[i] \equiv d_{001-\mathrm{IF}}^{110-\square}[i]$.

$$
d_{110-\mathrm{IF}}^{110-\square}[i]=a_{\mathrm{UC}}\left(i+\frac{3}{4}\right) .
$$

The height of the rectangular cross section follows straightforwardly from $h[i] \equiv d_{110-\mathrm{IF}}^{110-\square}[i]$.

$$
A^{110-\square_{[i]}}=\left(a_{\mathrm{UC}}\right)^{2} \frac{(i+1)(4 i+3)}{4(\sqrt{2})} \text {. }
$$

The cross section of this NWire type is shown in Fig. 3.

3.3. NWires growing along the [110] direction with hexagonal cross section and four $\{111\}$ plus two $\{001\}$ interfaces

The remaining four NWire types to be investigated all have a hexagonal cross section which has a more complex geometry. Owing to the fact that each hexagonal cross-section type has two different configurations, namely a different configuration at the central point, we present an even and an odd series for each NWire type. We will see in Section 4.2 that there is a real 


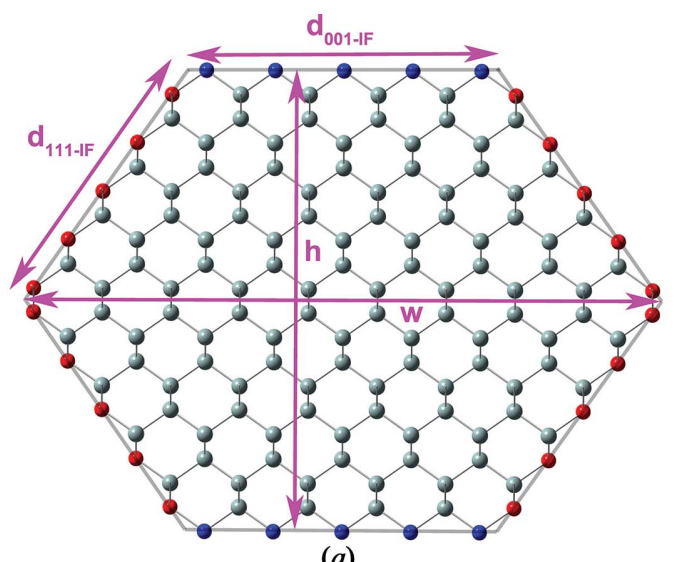

(a)
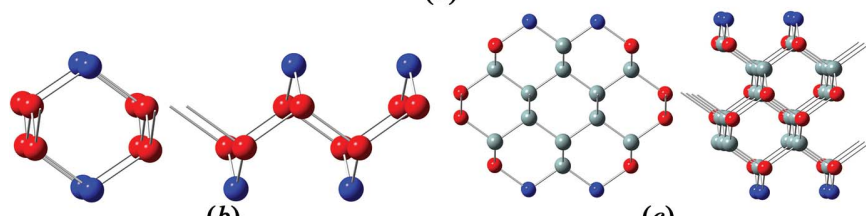

(b)
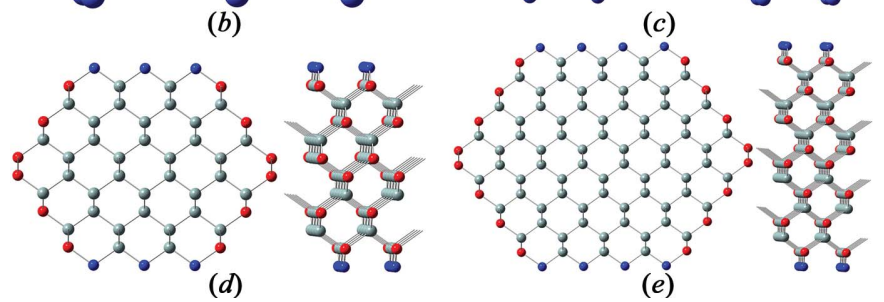

Figure 4

(a) Definitions of the characteristic lengths for the $\mathrm{zb}$ - and diamondstructured NWires growing along the [110] axis with a hexagonal cross section and two $\{001\}$ and four $\{111\}$ interfaces, which are shown by translucent black lines. $(b)-(e)$ Top and side views of the first four members $(i=1$ to 4$)$ of the even series. (b) $\mathrm{X}_{12},(c) \mathrm{X}_{48},(d) \mathrm{X}_{108}$ and (e) $\mathrm{X}_{192}$. For atom colours see Fig. 3.

need for such detailed descriptions when evaluating HR-TEM images.

$$
\begin{gathered}
N_{\text {Wire,even }}^{110-\square}[i]=12 i^{2}, \\
N_{\text {bnd,even }}^{110-\square}[i]=8 i(3 i-1), \\
N_{\mathrm{IF}, \text { even }}^{110-\square}[i]=16 i, \\
\frac{N_{111-\mathrm{IF}, \text { even }}^{110-\square}[i]}{N_{001-\mathrm{IF}, \text { even }}^{110}[i]}=1 \forall i,
\end{gathered}
$$

From equations (16) and (17), it follows straightforwardly that $N_{111-\mathrm{IF} \text {,even }}^{110-\square}[i]=N_{001-\mathrm{IF} \text {,even }}^{110-\square}[i]=16 i$.

$$
\begin{gathered}
d_{001-\mathrm{IF}, \text { even }}^{110-\mathrm{O}}[i]=a_{\mathrm{UC}} \sqrt{\frac{1}{8}}(2 i-1), \\
d_{111-\mathrm{IF}, \text { even }}^{110-\square}[i]=a_{\mathrm{UC}} \sqrt{\frac{3}{8}}\left(i-\frac{1}{4}\right), \\
w_{\text {even }}^{110-\bigcirc}[i]=a_{\mathrm{UC}} \sqrt{2}\left(i-\frac{3}{8}\right),
\end{gathered}
$$

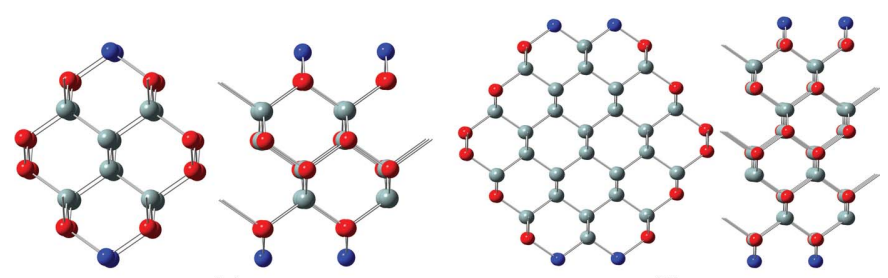

(a)

(b)

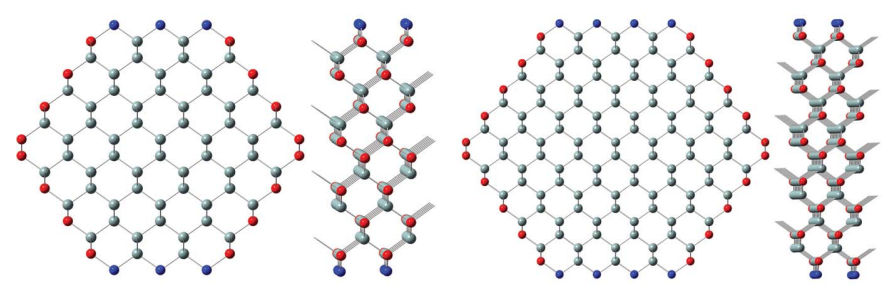

(c)

(d)

Figure 5

Cross sections and side views of zb- and diamond-structured NWires growing along the [110] axis with a hexagonal cross section and two $\{001\}$ and four $\{111\}$ interfaces, odd series, for run indices $i=1$ to 4 . (a) $\mathrm{X}_{32},(b)$ $\mathrm{X}_{84},(c) \mathrm{X}_{160}$ and $(d) \mathrm{X}_{260}$. For atom colours and definitions of characteristic lengths, see Figs. 3 and 4, respectively.

$$
\begin{gathered}
h_{\text {even }}^{110-\bigcirc}[i]=a_{\mathrm{UC}}\left(i-\frac{1}{4}\right), \\
A_{\text {even }}^{110-\bigcirc}[i]=\left(a_{\mathrm{UC}}\right)^{2} \sqrt{\frac{1}{8}}\left(3 i-\frac{5}{4}\right)\left(i-\frac{1}{4}\right) .
\end{gathered}
$$

The cross section of this even NWire type is shown in Fig. 4, together with the definitions of the characteristic lengths.

For the odd series (Fig. 5) we get

$$
\begin{gathered}
N_{\text {Wire,odd }}^{110-\square}[i]=4(i[3 i+4]+1), \\
N_{\text {bnd,odd }}^{110-\square}[i]=4(6 i[i+1]+1) \\
N_{\mathrm{IF}, \text { odd }}^{110-\square}[i]=8(2 i+1),
\end{gathered}
$$

whereby

$$
\frac{N_{11-\mathrm{IF}, \text { odd }}^{110-}[i]}{N_{001-\mathrm{IF}, \text { odd }}^{110}[i]}=\frac{8(i+1)}{8 i}=1+\frac{1}{i} .
$$

Again, the central expression in equation (26) shows both number series in their explicit form, while the right-hand expression presents the simplified result of their ratio.

$$
d_{001-\mathrm{IF}, \mathrm{odd}}^{110-\mathrm{O}}[i]=a_{\mathrm{UC}} \sqrt{\frac{1}{8}}(2 i-1) .
$$

Comparing this result with equation (18), we see that $d_{001-\mathrm{IF}, \text { odd }}^{110-Q}[i]=d_{001-\mathrm{IF}, \text { even }}^{110-\mathrm{C}}[i]$.

$$
d_{111-\mathrm{IF}, \mathrm{odd}}^{110-}[i]=a_{\mathrm{UC}} \sqrt{\frac{3}{8}}\left(i+\frac{3}{4}\right) .
$$

Comparing this result with equation (19), we see that $d_{111-\mathrm{IF}, \text { odd }}^{110}[i]=d_{111-\mathrm{IF}, \text { even }}^{110-\bigcirc}[i+1]$.

$$
w_{\mathrm{odd}}^{110-}[i]=a_{\mathrm{UC}} \sqrt{2}\left(i+\frac{1}{8}\right) .
$$


The relation to the even series is $w_{\text {odd }}^{110-\square}[i]=w_{111-\mathrm{IF} \text {, even }}^{110-\square}\left[i+\frac{1}{2}\right]$.

$$
h_{\mathrm{odd}}^{110-\bigcirc}[i]=a_{\mathrm{UC}}\left(i+\frac{3}{4}\right) \text {. }
$$

This result differs from the even series by one $a_{\mathrm{UC}} ; h_{\text {odd }}^{110-\square}[i]=$ $h_{111-\mathrm{IF}, \text { even }}^{110-\square}[i+1]$.

$$
A_{\text {odd }}^{110-\bigcirc}[i]=\left(a_{\mathrm{UC}}\right)^{2} \sqrt{\frac{1}{8}}\left(3 i-\frac{1}{4}\right)\left(i+\frac{3}{4}\right) .
$$

3.4. NWires growing along the $[11 \overline{2}]$ direction with hexagonal cross section and four $\{1 \overline{3} 1\}$ plus two $\{111\}$ interfaces

$$
\begin{gathered}
N_{\text {Wire,even }}^{11 \overline{1}-\bigcirc}[i]=4 i(3 i+1), \\
N_{\text {bnd,even }}^{11 \overline{2}-\bigcirc}[i]=2\left(12 i^{2}-1\right), \\
N_{\mathrm{IF}, \text { even }}^{11 \overline{2}-\bigcirc}[i]=16\left(i+\frac{1}{4}\right) .
\end{gathered}
$$

The assignment of interface bonds to the $\{111\}$ and $\{1 \overline{3} 1\}$ planes is shown in Fig. 6(a). With this assignment of interface atoms to the $\{111\}$ and $\{1 \overline{3} 1\}$ planes we obtain

$$
\frac{N_{1 \overline{3} 1-\mathrm{IF}, \text { even }}^{11 \overline{2}-\mathrm{C}}[i]}{N_{111-\mathrm{IF}, \text { even }}^{11 \overline{2}-\square}[i]}=\frac{4(3 i+2)}{4(i-1)}=\frac{3 i+2}{i-1} .
$$

As before, the central expression in equation (35) shows both number series in their explicit form, while the right-hand expression presents the simplified result of their ratio.

$$
\begin{gathered}
d_{111-\mathrm{IF}, \mathrm{even}}^{11 \overline{2}-\bigcirc}[i]=a_{\mathrm{UC}} \sqrt{\frac{1}{8}}(2 i-1), \\
d_{1 \overline{3} 1-\mathrm{IF}, \mathrm{even}}^{11 \overline{2}-\bigcirc}[i]=a_{\mathrm{UC}} \sqrt{\frac{11}{24}} i, \\
w_{\mathrm{even}}^{11 \overline{2}-\bigcirc}[i]=a_{\mathrm{UC}} \sqrt{\frac{1}{8}}(4 i-1), \\
h_{\mathrm{even}}^{11 \overline{2}-\bigcirc}[i]=a_{\mathrm{UC}} \sqrt{\frac{4}{3}} i, \\
A_{\mathrm{even}}^{11 \overline{2}-\bigcirc}[i]=\left(a_{\mathrm{UC}}\right)^{2} \sqrt{\frac{1}{6}} i(3 i-1) .
\end{gathered}
$$

For the odd series (Fig. 6) we get

$$
\begin{gathered}
N_{\text {Wire,odd }}^{11 \overline{2}-\square}[i]=12 i(i+2)+10, \\
N_{\text {bnd,odd }}^{11 \overline{2}-\bigcirc}[i]=2(6 i+1)(2 i+3)+5, \\
N_{\mathrm{IF}, \text { odd }}^{11 \overline{-}-\bigcirc}[i]=2(8 i+9),
\end{gathered}
$$

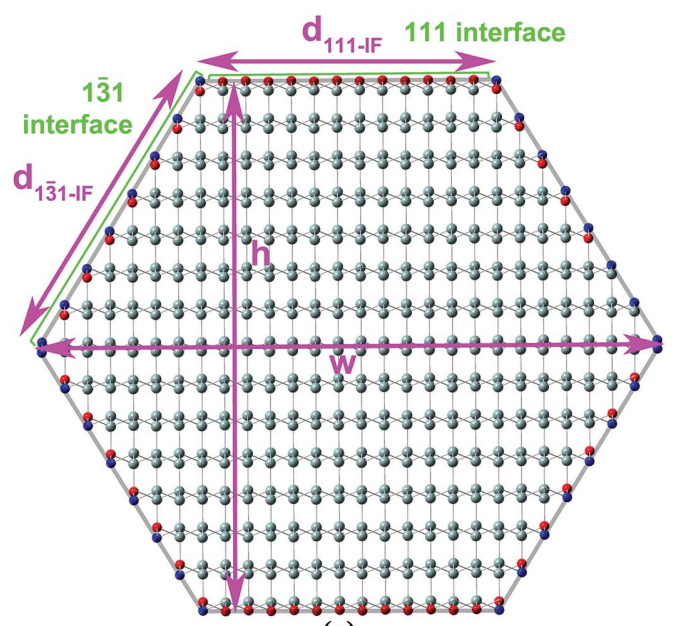

(a)

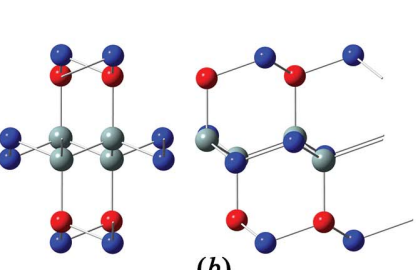

(b)

8

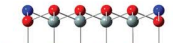

8939398

$8.8+8 \times 8+88$

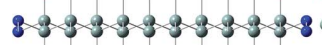

89898988

$2+3.9+8.8$

89888

Figure 6

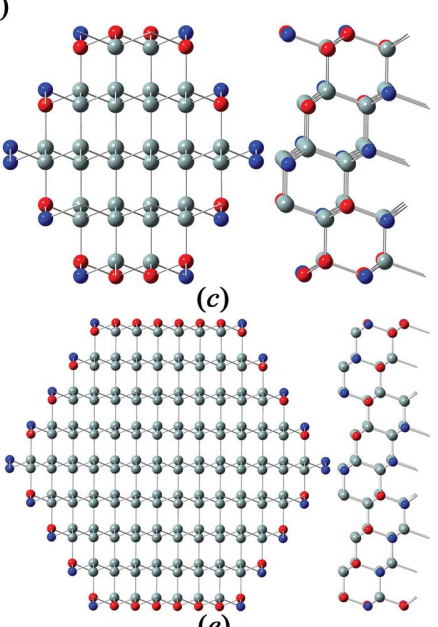

(e) (a) Definitions of characteristic lengths for the $\mathrm{zb}$ - and diamond-

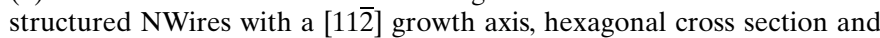
four $\{11 \overline{3}\}$ plus two $\{111\}$ interfaces, which are shown by translucent black lines. Green markings show the assignment of interface atoms (red or blue) to the respective interface orientation in accordance with the number series for interface bonds $N_{\mathrm{IF}}^{11 \overline{2}-} \square_{[i]}$. (b)-(e) Top and side views of the first four members $(i=1$ to 4$)$ of the even series. (b) $\mathrm{X}_{16},(c) \mathrm{X}_{56},(d)$ $\mathrm{X}_{120}$ and $(e) \mathrm{X}_{208}$. For atom colours see Fig. 3.

$$
\frac{N_{1 \overline{3} 1-\mathrm{IF}, \text { odd }}^{11 \overline{2}-\square}[i]}{N_{111}^{11 \overline{2}-\mathrm{IF}, \text { odd }}[i]}=\frac{4(3 i+5)}{2(2 i-1)}=\frac{2(3 i+5)}{2 i-1} .
$$

Again, the central expression of equation (44) shows both number series in their explicit form, while the right-hand expression presents the simplified result of their ratio.

$$
d_{111-\mathrm{IF}, \mathrm{odd}}^{11 \overline{2}-\mathrm{Q}}[i]=a_{\mathrm{UC}} \sqrt{\frac{1}{2}} i
$$

$$
\begin{gathered}
d_{1 \overline{3} 1-\mathrm{IF}, \mathrm{odd}}^{11 \overline{2}-\bigcirc}[i]=a_{\mathrm{UC}} \sqrt{\frac{11}{24}}(i+1), \\
w_{\mathrm{odd}}^{11 \overline{2}-\bigcirc}[i]=a_{\mathrm{UC}} \sqrt{\frac{1}{2}}(2 i+1),
\end{gathered}
$$




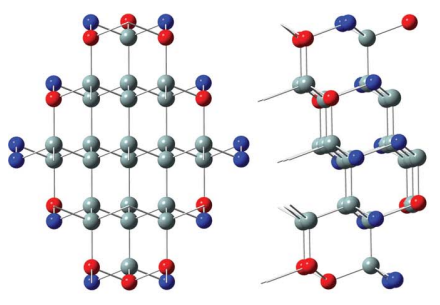

(a)

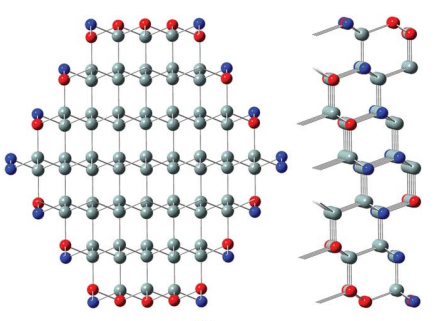

(b)

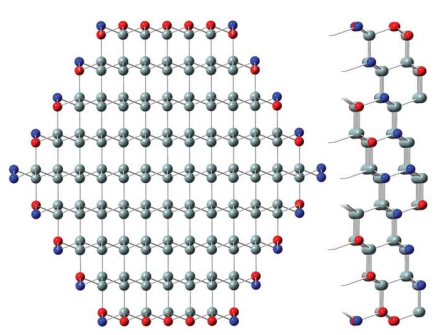

(c)

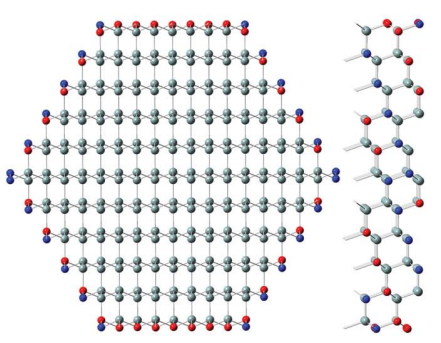

(d)
Figure 7

Cross sections and side views of $\mathrm{zb}$ - and diamond-structured NWires with

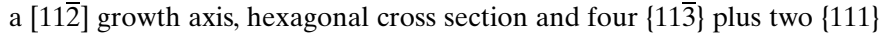
interfaces, odd series, for run indices $i=1$ to 4 . (a) $\mathrm{X}_{46},(b) \mathrm{X}_{106},(c) \mathrm{X}_{190}$ and $(d) \mathrm{X}_{298}$. For atom colours and definitions of the characteristic lengths and interfaces, see Figs. 3 and 6, respectively.

$$
\begin{gathered}
h_{\mathrm{odd}}^{11 \overline{2}-} \bigcirc[i]=a_{\mathrm{UC}} \sqrt{\frac{4}{3}}(i+1), \\
A_{\mathrm{odd}}^{11 \overline{2}-\bigcirc}[i]=\left(a_{\mathrm{UC}}\right)^{2} \sqrt{\frac{1}{6}}(3 i+1)(i+1) .
\end{gathered}
$$

The cross section for this odd series NWire type is shown in Fig. 7.

3.5. NWires growing along the [111] direction with hexagonal cross section and six $\{110\}$ interfaces

$$
\begin{gathered}
N_{\text {Wire,even }}^{111-\bigcirc \mid 110}[i]=6 i(i+1)+2, \\
N_{\text {bnd,even }}^{111-\bigcirc \mid 110}[i]=6 i(2 i+1)+1, \\
N_{\mathrm{IF}, \text { even }}^{111-\bigcirc \mid 110}[i]=6(2 i+1), \\
d_{\mathrm{IF}, \text { even }}^{111-\bigcirc \mid 110}[i]=a_{\mathrm{UC}} \frac{\sqrt{11}}{8} i, \\
w_{\text {even }}^{111-\mathrm{O} \mid 110}[i]=a_{\mathrm{UC}} \frac{\sqrt{11}}{4} i .
\end{gathered}
$$

For a regular hexagon with $60^{\circ}$ rotational symmetry, we get $w_{\text {even }}^{111-\mathrm{O} \mid 110}[i]=2 d_{\mathrm{IF} \text {, even }}^{111-\bigcirc \mid 110}[i]$.

$$
\begin{gathered}
h_{\text {even }}^{111-{ }^{110}}[i]=a_{\mathrm{UC}} \frac{1}{\sqrt{2}} i, \\
A_{\text {even }}^{111-\square \mid 110}[i]=\left(a_{\mathrm{UC}}\right)^{2} \frac{3}{8} \sqrt{\frac{11}{8}} i^{2} .
\end{gathered}
$$

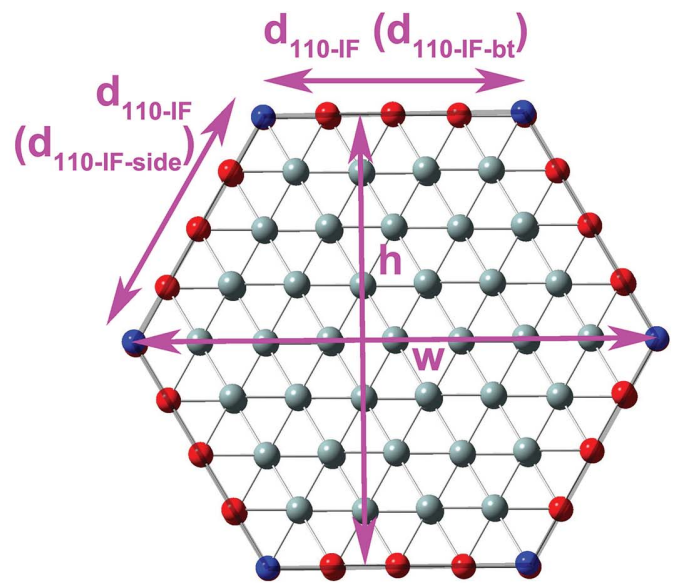

(a)

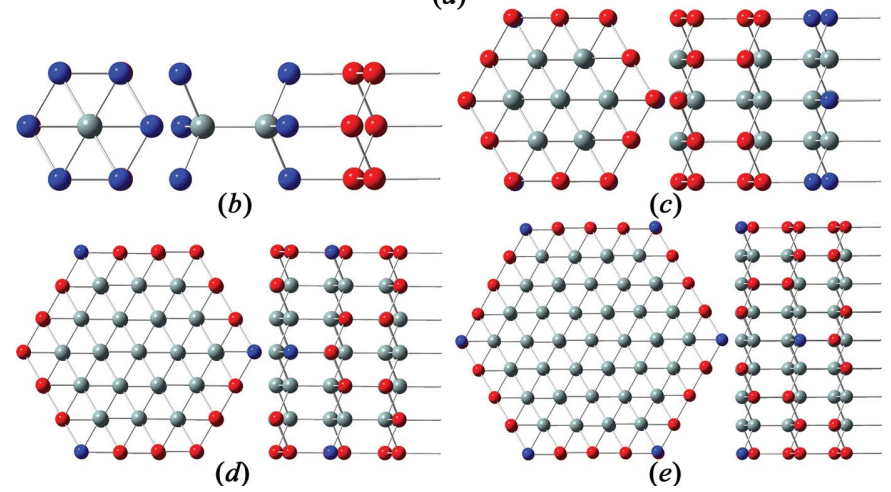

Figure 8

(a) Definitions of characteristic lengths for the zb- and diamondstructured NWires with a [111] growth axis, hexagonal cross section and $\{110\}$-oriented interfaces, which are shown by translucent black lines. Parameters in brackets refer to the odd cross-section series where the interface lengths are not equal (see Fig. 9). (b)-(e) Top and side views of the first four members $(i=1$ to 4$)$ of the even series. $(b) \mathrm{X}_{14},(c) \mathrm{X}_{38},(d)$ $\mathrm{X}_{74}$ and $(e) \mathrm{X}_{122}$. For atom colours see Fig. 3 .

Fig. 8 shows the cross section of this even NWire type, together with the definitions of the characteristic lengths.

For the odd series, we obtain

$$
\begin{gathered}
N_{\text {Wire,odd }}^{111-\text { O } \mid 110}[i]=2 i(3 i+7)+8, \\
N_{\text {bnd,odd }}^{111-\text { O } \mid 110}[i]=2 i(6 i+11)+9, \\
N_{\mathrm{IF}, \text { odd }}^{111-\square \mid 110}[i]=2(6 i+7), \\
d_{\mathrm{IF}, \text { odd,tb }}^{111-\mathrm{O} \mid 110}[i]=a_{\mathrm{UC}} \frac{\sqrt{11}}{8} i .
\end{gathered}
$$

The subscript tb stands for the top and bottom interfaces as illustrated in Fig. 8. This result is equal to $d_{\mathrm{IF} \text {,even }}^{111-\mathrm{O} \mid 110}[i]$.

$$
d_{\mathrm{IF}, \text { odd, side }}^{111-\mathrm{Q} \mid 110}[i]=a_{\mathrm{UC}} \frac{\sqrt{11}}{8}(i+1) .
$$

We see that $d_{\mathrm{IF}, \text { odd,side }}^{111-\bigcirc \mid 110}[i]=d_{\mathrm{IF}, \text { even }}^{111-\bigcirc \mid 110}[i+1]$.

$$
w_{\mathrm{odd}}^{111-\mathrm{O} \mid 110}[i]=a_{\mathrm{UC}} \frac{\sqrt{11}}{4}\left(i+\frac{1}{2}\right) \text {. }
$$

Here, the relation to the even series is $w_{\text {odd }}^{111-0 \mid 110}[i]=$ $w_{\text {even }}^{111-\square \mid 110}\left[i+\frac{1}{2}\right]$. 


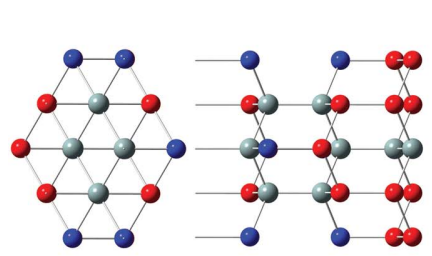

(a)

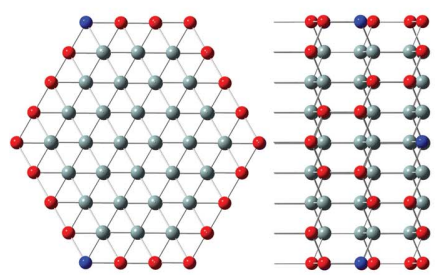

(c)

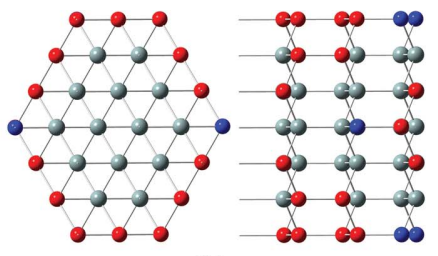

(b)

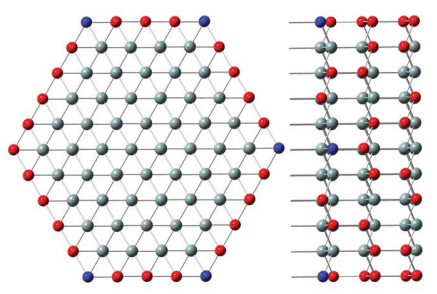

(d)

Figure 9

Cross sections and side views of $\mathrm{zb}$ - and diamond-structured NWires with a [111] growth axis, hexagonal cross section and $\{110\}$-oriented interfaces, odd series, for run indices $i=1$ to 4. (a) $\mathrm{X}_{28},(b) \mathrm{X}_{60},(c) \mathrm{X}_{104}$ and $(d) \mathrm{X}_{160}$. For atom colours and definitions of the characteristic lengths, see Figs. 3 and 8 , respectively.

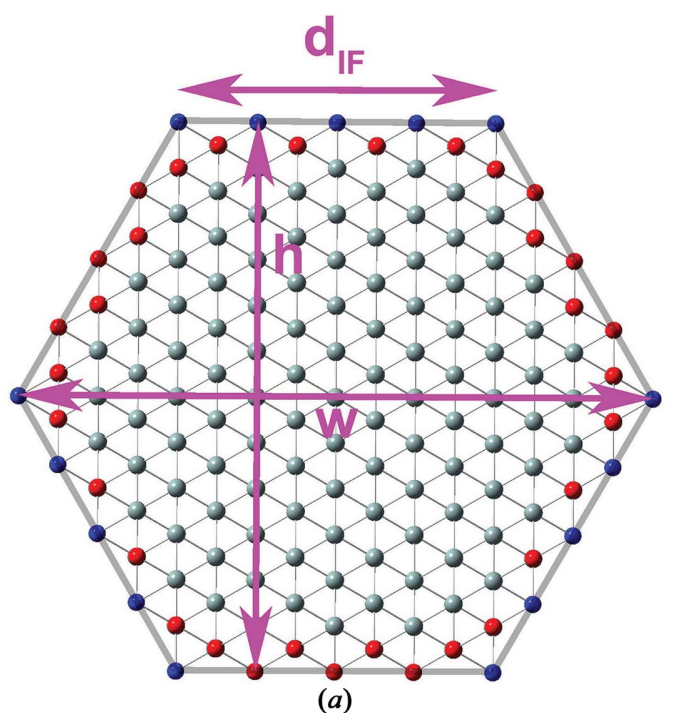

(a)

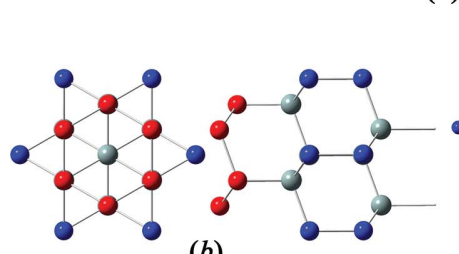

(b)

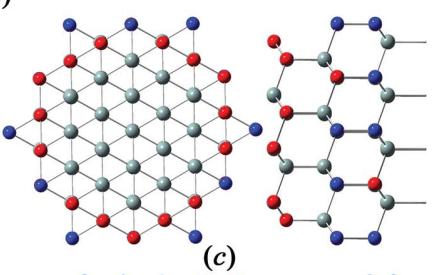

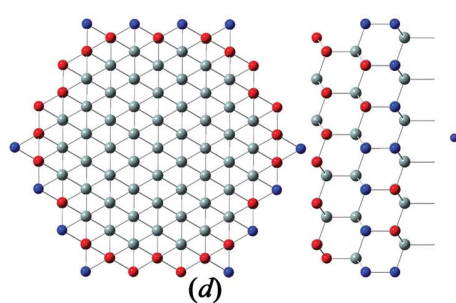

Figure 10

(a) Definitions of the characteristic lengths for the even series of zb- and diamond-structured NWires with a [111] growth axis and hexagonal cross section. All interfaces have $\{11 \overline{2}\}$ orientation, shown by translucent black lines. For definitions of the odd series see Fig. 11(a). (b)-(e) Top and side views of the first four members ( $i=1$ to 4$)$ of the even series. (b) $\mathrm{X}_{26},(c)$ $\mathrm{X}_{86},(d) \mathrm{X}_{182}$ and $(e) \mathrm{X}_{314}$. For atom colours see Fig. 3.

$$
h_{\mathrm{odd}}^{111-\mathrm{D} \mid 110}[i]=a_{\mathrm{UC}} \frac{1}{\sqrt{2}}(i+1) .
$$

Again, this result relates to the even series via $h_{\text {odd }}^{111-0 / 110}[i]=$ $h_{\text {even }}^{111-0 \mid 110}[i+1]$.

$$
A_{\mathrm{odd}}^{111-\mathrm{O} \mid 110}[i]=\left(a_{\mathrm{UC}}\right)^{2} \frac{1}{8} \sqrt{\frac{11}{8}}(3 i+1)(i+1) .
$$

The cross section of this odd NWire type is shown in Fig. 9.

3.6. NWires growing along the [111] direction with a

hexagonal cross section and six $\{11 \overline{2}\}$ interfaces

$$
\begin{gathered}
N_{\text {Wire,even }}^{111-\mathrm{Q} \mid 11 \overline{2}}[i]=6 i(3 i+1)+2, \\
N_{\text {bnd,even }}^{111-\bigcirc \mid 11 \overline{2}}[i]=(6 i)^{2}+1, \\
N_{\mathrm{IF}, \text { even }}^{111-\square \mid 11 \overline{2}}[i]=6(4 i+1), \\
d_{\mathrm{IF}, \text { even }}^{111-\bigcirc \mid 11 \overline{2}}[i]=a_{\mathrm{UC}} \frac{1}{\sqrt{2}} i, \\
w_{\text {even }}^{111-\mathrm{Q} \mid 11 \overline{2}}[i]=a_{\mathrm{UC}} \sqrt{2} i .
\end{gathered}
$$

For a regular hexagon with $60^{\circ}$ rotational symmetry, we get $w_{\text {even }}^{111-\square \mid 11 \overline{2}}[i]=2 d_{\mathrm{IF}, \text { even }}^{111-\square \mid 11 \overline{2}}[i]$.

$$
\begin{gathered}
h_{\text {even }}^{111-\mathrm{D} \mid 11 \overline{2}}[i]=a_{\mathrm{UC}} \frac{3}{8} \sqrt{11} i, \\
A_{\text {even }}^{111-\mathrm{Q} \mid 11 \overline{2}}[i]=\left(a_{\mathrm{UC}}\right)^{2}\left(\frac{3}{4}\right)^{2} \sqrt{\frac{11}{2}} i^{2} .
\end{gathered}
$$

The cross section of this even NWire type is shown in Fig. 10, together with the definitions of the characteristic lengths.

For the odd series, we get

$$
\begin{gathered}
N_{\text {Wire,odd }}^{111-\square \mid 11 \overline{2}}[i]=2 i(9 i+7)+4, \\
N_{\text {bnd,odd }}^{111-\square \mid 11 \overline{2}}[i]=4 i(9 i+4)+3, \\
N_{\mathrm{IF}, \text { odd }}^{111-\square \mid 11 \overline{2}}[i]=2(12 i+5) . \\
d_{\mathrm{IF}, \text { odd,tb }}^{111-\square \mid 11 \overline{2}}[i]=a_{\mathrm{UC}} \frac{1}{\sqrt{2}} i .
\end{gathered}
$$

Comparison with equation (68) yields $d_{\mathrm{IF}, \text { odd, tb }}^{111-\mathrm{D} \mid 11 \overline{2}}[i]=$ $d_{\mathrm{IF}, \text { even }}^{111-\bigcirc \mid 11 \overline{2}}[i]$.

$$
d_{\mathrm{IF}, \text { odd,side }}^{111-\mathrm{D} \mid 11 \overline{2}}[i]=a_{\mathrm{UC}} \frac{1}{\sqrt{2}}\left[i+\sqrt{\frac{11}{96}}\right] .
$$

This result differs from $d_{\mathrm{IF}, \text { odd,tb }}^{111-\mathrm{D} \mid 11 \overline{2}}[i]$ by the offset of $\sqrt{11 / 96}$ on $i$ due to the corners between the $\{11 \overline{2}\}$ interfaces (see Fig. 11).

$$
w_{\mathrm{odd}}^{111-\mathrm{O} \mid 11 \overline{2}}[i]=a_{\mathrm{UC}} \sqrt{2}\left(i+\frac{1}{2} \sqrt{\frac{11}{96}}\right) .
$$




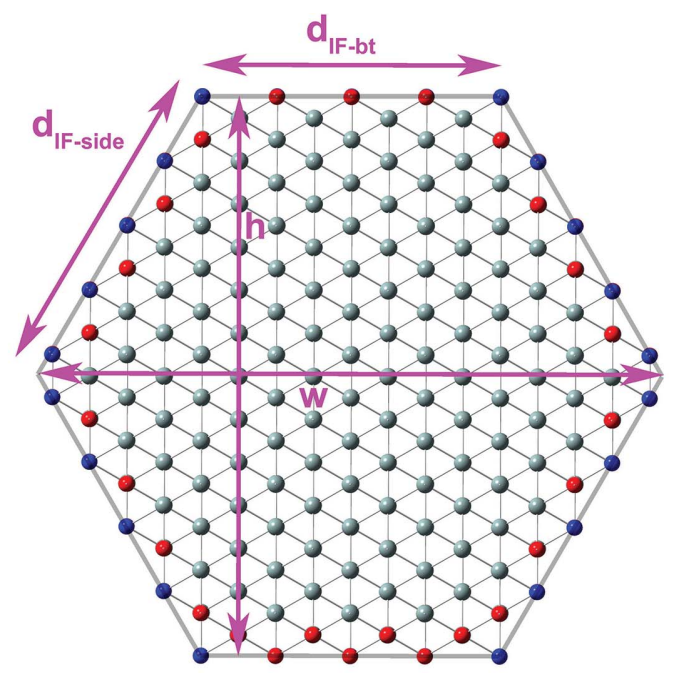

(a)

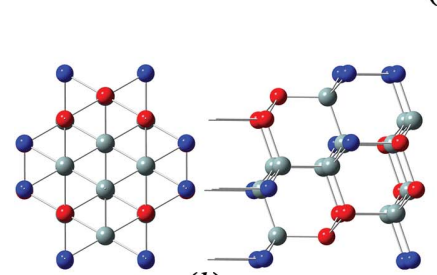

(b)

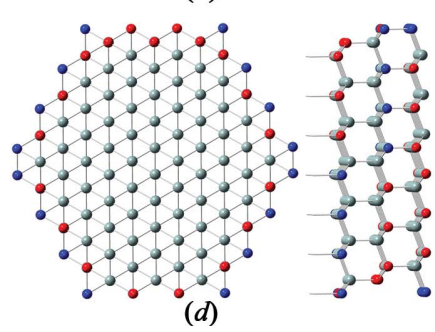

Figure 11

(a) Definitions of the characteristic lengths for the odd series of zb- and diamond-structured NWires growing along the [111] axis with a hexagonal cross section and all interfaces with $\{11 \overline{2}\}$ orientation, shown by translucent black lines. $(b)-(e)$ Top and side views of the first four members $(i=1$ to 4$)$ of the odd series. (b) $\mathrm{X}_{36},(c) \mathrm{X}_{104},(d) \mathrm{X}_{208}$ and $(e)$ $\mathrm{X}_{348}$. For atom colours see Fig. 3.

The corners between the $\{11 \overline{2}\}$ interfaces are reflected in an offset of the run index compared with the even series so that $w_{\text {odd }}^{111-\square \mid 11 \overline{2}}[i]=w_{\text {even }}^{111-\bigcirc \mid 11 \overline{2}}\left[i+\frac{1}{2} \sqrt{11 / 96}\right]$.

$$
\begin{gathered}
h_{\mathrm{odd}}^{111-\square \mid 11 \overline{2}}[i]=a_{\mathrm{UC}} \frac{\sqrt{11}}{8}(3 i+1), \\
A_{\mathrm{odd}}^{111-\bigcirc \mid 11 \overline{2}}[i]=\left(a_{\mathrm{UC}}\right)^{2} \frac{9}{8} \sqrt{\frac{11}{8}}\left(i+\frac{\sqrt{33}}{48}\right)\left(i+\frac{1}{3}\right) .
\end{gathered}
$$

The cross section of this odd NWire type is shown in Fig. 11, together with the definitions of the characteristic lengths.

\section{Application examples of nanowire cross sections}

\subsection{Morphological description of III-V nanowires}

III-V NWires are often found to grow along the [111] axis, which requires the least energy (see Table 1), with hexagonal
Table 5

Structural parameters for the even series of hexagonal cross sections with [111] growth and $\{11 \overline{2}\}$ interfaces with $i=180$ (Fig. 10) and for the even series of hexagonal cross sections with [111] growth and $\{110\}$ interfaces with $i=394$ (Fig. 8); the slab thickness of the NWire cross section is $d_{\text {slab }}=$ $a_{\mathrm{UC}} \sqrt{3}$ in all cases.

\begin{tabular}{lllll}
\hline & $\begin{array}{l}111-\square \mid 11 \overline{2} \\
{[i=180]}\end{array}$ & $\begin{array}{l}111-\square \mid 110 \\
{[i=394]}\end{array}$ & $\begin{array}{l}111-\square \mid 110 \\
{[i=461]}\end{array}$ & $\begin{array}{l}111-\mathrm{O} \mid 110 \\
\mathrm{Al}_{0.5} \mathrm{In}_{0.5} \mathrm{P} \\
\text { shell }\end{array}$ \\
\hline $\begin{array}{l}\text { Parameter } \\
N_{\text {Wire }} / d_{\text {slab }}\end{array}$ & 584282 & 933782 & 1277894 & 344112 \\
$N_{\text {bnd }} / d_{\text {slab }}$ & 1166401 & 1865197 & 2553019 & 678348 \\
$N_{\text {IF }} / d_{\text {slab }}$ & 4326 & 4734 & 5538 & 10272 \\
$d_{\text {IF }}(\mathrm{nm})$ & 71.95 & 92.34 & 108.0 & \\
$w(\mathrm{~nm})$ & 143.9 & 184.7 & 216.1 & \\
$w_{\text {shell }}(\mathrm{nm})$ & & & & 13.40 \\
$h(\mathrm{~nm})$ & 126.6 & 157.5 & 184.3 & \\
$\left.A(\mathrm{~nm})^{2}\right)$ & 13660 & 21816 & 29867 & 7939.7 \\
$A_{\mathrm{IF}}\left(\mathrm{nm}^{2}\right)$ & & & & 110.88 \\
$N_{\text {bnd }} / N_{\text {Wire }}$ & 1.9963 & 1.9975 & 1.9978 & 1.9713 \\
$N_{\mathrm{IF}} / N_{\text {Wire }}$ & $7.404 \times 10^{-3}$ & $5.070 \times 10^{-3}$ & $4.334 \times 10^{-3}$ & $2.985 \times 10^{-2}$ \\
$N_{\text {IF }} / N_{\text {bnd }}$ & $3.709 \times 10^{-3}$ & $2.538 \times 10^{-3}$ & $2.169 \times 10^{-3}$ & $1.514 \times 10^{-2}$ \\
\hline
\end{tabular}

cross sections (Joyce et al., 2011; Treu et al., 2015). Such NWires have experimental cross sections of $\{11 \overline{2}\}$ interfaces [Figs. 12(a) and 12(b) (Sköld et al., 2006)] or $\{110\}$ interfaces [Figs. 12(c) and 12(d) (Sköld et al., 2006)]. Below, we will show the structural parameters we can derive using equations (68) and (53) to match the side lengths and obtain the respective run index $i$.

Fig. 12(c) shows a GaAs core with a hexagonal cross section, surrounded by an $\mathrm{Al}_{0.5} \mathrm{In}_{0.5} \mathrm{P}$ shell visible as a bright hexagonal ring. We focus on the GaAs partition of both NWires. The scale bars in those images represent $100 \mathrm{~nm}$ and allow an estimation of the side lengths of the hexagonal cross section, which are $72 \mathrm{~nm}$ for the $\{11 \overline{2}\}$ interfaces and $92.4 \mathrm{~nm}$ for the $\{110\}$ interfaces. With $a_{\mathrm{uc}}(\mathrm{GaAs})=0.56533 \mathrm{~nm}$ (Böer, $1990)$, these side lengths match $d_{\mathrm{IF}, \text { even }}^{111-\mathrm{Q} 11 \overline{2}}[i=180]=$ $a_{\mathrm{UC}} 180 / \sqrt{2}=71.95 \mathrm{~nm}$ and $d_{\mathrm{IF}, \text { even }}^{111-\bigcirc \mid 110}[i=394]=a_{\mathrm{UC}} \times 394$ $\times \sqrt{11} / 8=92.34 \mathrm{~nm}$. As the even and odd series converge in their parameters for large $i$, the use of the latter series does not yield more accurate information unless structural information around the centre point of the cross section is available; see Fig. 13 for an example. Data obtained from $i=180$ for hexagonal cross sections with [111] growth and $\{11 \overline{2}\}$ interfaces and from $i=394$ for hexagonal cross sections with [111] growth and $\{110\}$ interfaces are listed in Table 5.

Analytical number series of the same cross-section type can be combined in any superposition seen fit to describe experimental data. We turn our attention to the NWire in Fig. 12(c) again to evaluate its complete morphology, i.e. a GaAs core with an $\mathrm{Al}_{0.5} \mathrm{In}_{0.5} \mathrm{P}$ shell, using our findings presented in Section 3.5. The shell has the same orientation as the GaAs core, growing in the [111] direction with $\{110\}$ interfaces. With the morphology of the entire core-shell NWire having a side length of $108 \mathrm{~nm}$ and monolithic lattice growth [Sköld et al., $\left.2006 ; \quad a_{\mathrm{UC}}\left(\mathrm{Al}_{0.5} \operatorname{In}_{0.4} \mathrm{P}\right) \simeq a_{\mathrm{UC}}(\mathrm{GaAs})\right]$, we arrive at $d_{\mathrm{IF}, \text { even }}^{111-\mathrm{O}}[110[i=461]$, from which it is straightforward to get all parameters as for the GaAs core (see Table 5). The parameters of the $\mathrm{Al}_{0.5} \mathrm{In}_{0.5} \mathrm{P}$ shell can be calculated by subtracting the parameters of the inner GaAs core from the parameters of 

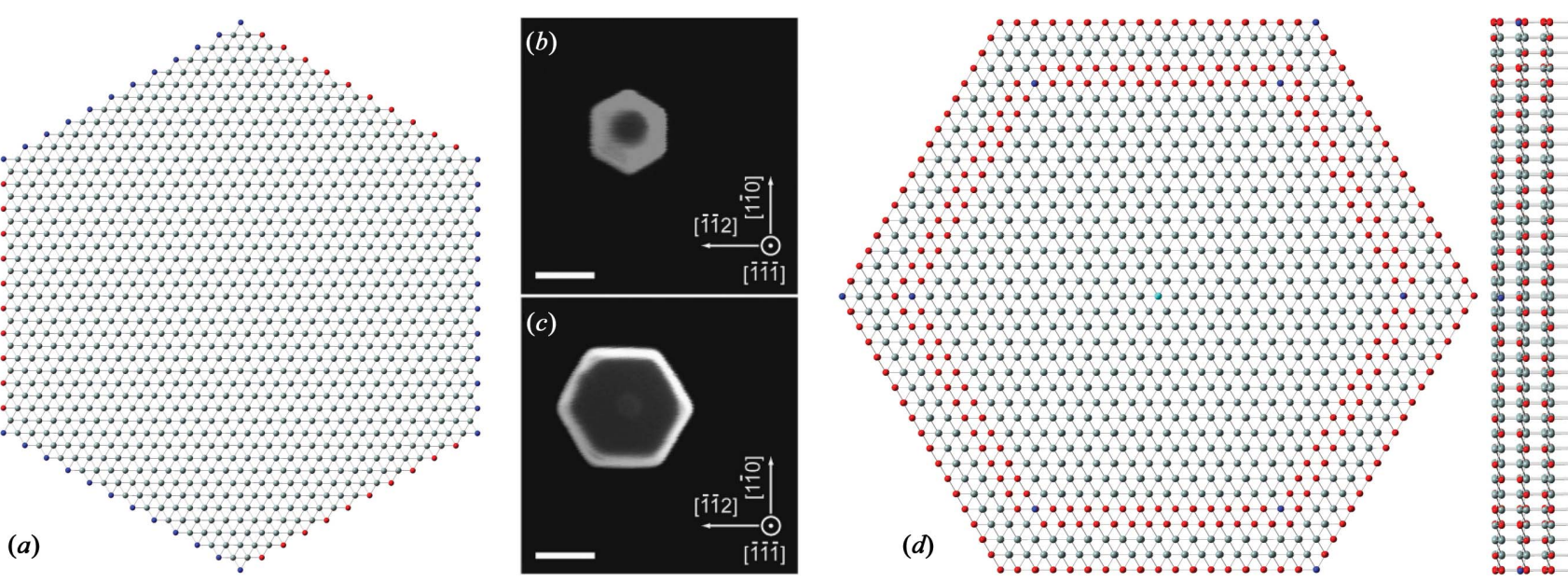

Figure 12

(a) Side and cross-sectional views of an $\mathrm{X}_{2246}(i=11)$ hexagonal NWire with a [111] growth axis and $\{11 \overline{2}\}$ interfaces. $(b)$ A cross-sectional STEM image of the same NWire type consisting of GaAs. Reprinted with permission from Sköld et al. (2006), copyright American Chemical Society 2006. (c) A crosssectional STEM image of a NWire with a [111] growth axis. The GaAs core shows as a dark hexagon and the AlInP shell is visible as a bright rim. Reprinted with permission from Sköld et al. (2006), copyright American Chemical Society 2006. The scale bars in panels $(b)$ and $(c)$ correspond to $100 \mathrm{~nm}$. (d) Side and cross-sectional views of a hexagonal NWire $\mathrm{X}_{2054}(i=18)$ by analogy with panel $(c)$, growing along the [111] axis with \{110\} interfaces and an internal $\{110\}$ interface separating the core from the shell. The symmetry centre is marked by a cyan atom. For other atom colours see Fig. 3.

the entire core-shell cross section. Due to the finite thickness of the interface layer, we choose the GaAs core parameters for the run index matching this core plus one $(i=394+1)$ to calculate the cross-section area of the $\mathrm{Al}_{0.5} \mathrm{In}_{0.5} \mathrm{P}$ shell: $A_{\mathrm{IF}}=$ $A_{\text {even }}^{111-\square \mid 110}[i=395]-A_{\text {even }}^{111-\square \mid 110}[i=394]$.

Along the same lines, we calculate the number of shell-internal bonds which is $N_{\text {bnd,even }}^{111-Q \mid 110}[i=461]-$ $N_{\text {bnd,even }}^{111-\bigcirc \mid 110}[i=395]$ and the cross-section area of the shell which is $A=A_{\text {even }}^{111-O \mid 110}[i=461]-A_{\text {even }}^{111-O \mid 110}[i=395]$.

The total number of interface bonds for the $\mathrm{Al}_{0.5} \operatorname{In}_{0.5} \mathrm{P}$ shell is the sum of its outer and internal interfaces to the GaAs core, yielding $N_{\mathrm{IF}}$ (shell) $=N_{\mathrm{IF} \text {,even }}^{111-\square \mid 110}[i=394]+N_{\mathrm{IF} \text {,even }}^{111-\bigcirc \mid 110}[i=461]$. The NWire cross section is a regular hexagon. Thus, simple trigonometry yields the thickness of the $\mathrm{Al}_{0.5} \mathrm{In}_{0.5} \mathrm{P}$ shell: $w_{\text {shell }}$ $=\Delta d_{\mathrm{IF}} /\left(2 \tan 30^{\circ}\right)=a_{\mathrm{UC}} \sqrt{33} / 16(461-395)$, with $\Delta d_{\mathrm{IF}}$ the difference in side lengths of the inner and outer hexagons defining the shell. All values are listed in Table 5.

We briefly look at some of the outcomes which can be derived from the values in Table 5 . As will be discussed in Section 5, the ratio of NWire-internal bonds per NWire atom $N_{\text {bnd }} / N_{\text {Wire }}$ is a sensitive stress indicator since fewer bonds per NWire atom are available to compensate external or internal stress. A good example of the latter is substitutional doping where the dopant, usually foreign to the NWire structure, causes significant amounts of strain. The values in Table 5 show that it should be either notably more difficult to dope the $\mathrm{Al}_{0.5} \mathrm{In}_{0.5} \mathrm{P}$ shell than the GaAs core, or that we can expect a significant rate of lattice defects introduced by the dopant. A quantitative statement will be given in Section 5 for Si NWires where experimental data for the increasing failure of impurity doping are available.

While the case above delivers valuable structural data, the full capacity of the analytical description of NWire cross sections comes to light when structural information on an

atomic basis is available, e.g. by HR-TEM. We will demonstrate these capabilities in Section 4.2.

\subsection{Morphological description of Si nanowires}

Si NWires are key candidates for future VLSI devices in sub-14 nm technology nodes (Weber \& Mikolajick, 2017). At

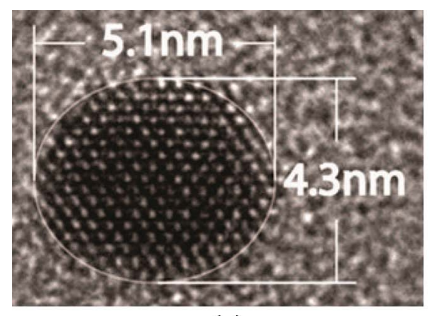

(a)

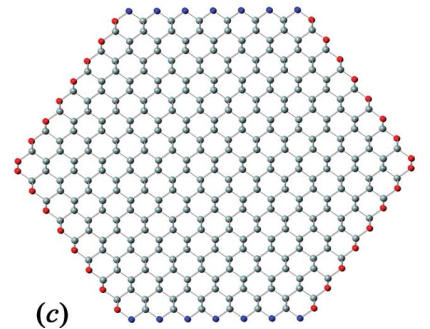

Figure 13

An ultrathin Si nanowire with a [110] axis embedded in $\mathrm{SiO}_{2}$, constituting a NWire FET structure. Reprinted with permission from Yi et al. (2011), copyright American Chemical Society 2011. Panel (a) shows the original image while panel $(b)$ includes the contours of the two analytical NWire cross sections. The red semi-translucent outline shows the odd series of the NWire growing along the [110] axis with $\{110\}$ and $\{001\}$ interfaces $(i=$ $\left.7, \mathrm{X}_{704}\right)$, as shown in panel $(c)$. The blue semi-translucent outline shows the same NWire type, but using its even series $\left(i=8, \mathrm{X}_{768}\right)$, as shown in panel $(d)$. For atom colours see Fig. 3. Details of the specific NWire cross sections shown in panels $(c)$ and $(d)$ are explained in Section 3.3. Additional structural information for both cross sections is listed in Table 6. 
Table 6

Structural parameters for hexagonal cross sections with a [110] growth axis, $\{111\}$ top and bottom interfaces and $\{001\}$ side interfaces of the odd series with $i=7$, and of the even series with $i=8$ (see Section 3.3 and Fig. 13); the slab thickness of the NWire cross sections is $d_{\text {slab }}=a_{\mathrm{UC}} \sqrt{2}$.

\begin{tabular}{|c|c|c|}
\hline Parameter & $i=7$, odd & $i=8$, even \\
\hline$N_{\text {Wire }} / d_{\text {slab }}$ & 704 & 768 \\
\hline$N_{\text {bnd }} / d_{\text {slab }}$ & 1348 & 1472 \\
\hline$N_{\mathrm{IF}} / d_{\text {slab }}$ & 120 & 128 \\
\hline$d_{001-\mathrm{IF}}^{110-\mathrm{O}}(\mathrm{nm})$ & 2.50 & 2.88 \\
\hline$d_{111-\mathrm{IF}}^{110-\bigcirc}(\mathrm{nm})$ & 2.58 & 2.58 \\
\hline$w^{110-} \bigcirc(\mathrm{nm})$ & 5.47 & 5.86 \\
\hline$h^{110-\square}(\mathrm{nm})$ & 4.21 & 4.21 \\
\hline$A^{110-\square}\left(\mathrm{nm}^{2}\right)$ & 16.76 & 18.38 \\
\hline$N_{\text {bnd }} / N_{\text {Wire }}$ & 1.915 & 1.917 \\
\hline$N_{\mathrm{IF}} / N_{\text {Wire }}$ & 0.170 & 0.167 \\
\hline$N_{\mathrm{IF}} / N_{\mathrm{bnd}}$ & 0.089 & 0.087 \\
\hline
\end{tabular}

such device dimensions, NWires have diameters significantly below $10 \mathrm{~nm}$, which allows us to image their entire cross sections at atomic resolution. This then provides the information to pick the right analytical description from Section 3 in terms of odd or even series. Often, such NWires follow a top-down approach by thermal oxidation combined with $\mathrm{SiO}_{2}$ back etching (Weber \& Mikolajick, 2017). Fig. 13(a) shows an HR-TEM image of such a NWire with a [110] axis orientation, $\{001\}$ interfaces on the top and bottom and $\{111\}$ side interfaces (Yi et al., 2011). This cross-section type was described in Section 3.3, and the nearest members of the respective odd and even series to fit the HR-TEM image are shown in Figs. 13(c) and 13(d). All structural parameters derived from the number series are listed in Table 6.

With these data, accurate structural metrology statements can be made for any characterization which has a structural dependence. The exact cross-section area allows us to calculate current densities. In contrast with absolute current values which are used in most work at present, current densities help us to interpret electronic phenomena such as impact ionization or inelastic carrier-carrier scattering. Thereby, the device characteristics of NWire-based electronic devices (Tomioka $e t$ al., 2011; Weber \& Mikolajick, 2017) can be assessed and interpreted in more detail.

\section{Usage of number series ratios on nanowire cross sections}

The ratio of intra-NWire bonds per NWire atom converges to 2 for NWire cross sections approaching infinity via their run index $i ; N_{\text {bnd }}[i] / N_{\text {Wire }}[i] \rightarrow 2$ for $i \rightarrow \infty$. This result follows directly from the consideration of the $\mathrm{zb}$ - or diamondstructured UC (Fig. 1). Every atom has four bonds to its first nearest neighbour (1-nn) atoms and each of these four bonds is shared with one of the 1-nn atoms. Depending on the size of the NWire, its interface faceting and its surface-to-volume ratio, $N_{\text {bnd }}[i] / N_{\text {Wire }}[i]$ can be significantly smaller than 2 .

In impurity doping, the dopants reside on substitutional lattice sites within the semiconductor such as $\mathrm{Si}$. This process requires a certain activation energy of which a significant part is taken up by neighbouring $\mathrm{Si}-\mathrm{Si}$ bonds, thereby inducing local stress. At very high dopant densities, the dopant solubility limit is reached, whereby many local doping events build up a global stress which either induces dopant clustering and interstitials or the disintegration of the semiconductor structure into its amorphous form. For nano-Si systems such as NWires or NCs, the ratio $N_{\text {bnd }}[i] / N_{\text {Wire }}[i]$ decreasing with system size reflects a decreasing ability of the nano-Si system to cope with local stress as induced by subsitutional doping.

This finding is reflected in the strong resistivity increase of Si NWires with shrinking diameter [Fig. 14(b)]. The argument of Björk et al. (2009) that dielectric mismatch with the enviroment prevents dopant ionization is certainly correct. However, dielectric mismatch is not the only cause of dopant failure. For Si NCs, their rejection of dopants to be located on substitutional lattice sites by self-purification has been discussed in detail (Dalpian \& Chelikowsky, 2006, 2008; König et al., 2015; Hiller et al., 2017a,b). Similar limits exist for Si NWires, as discussed below. Even for doping densities of
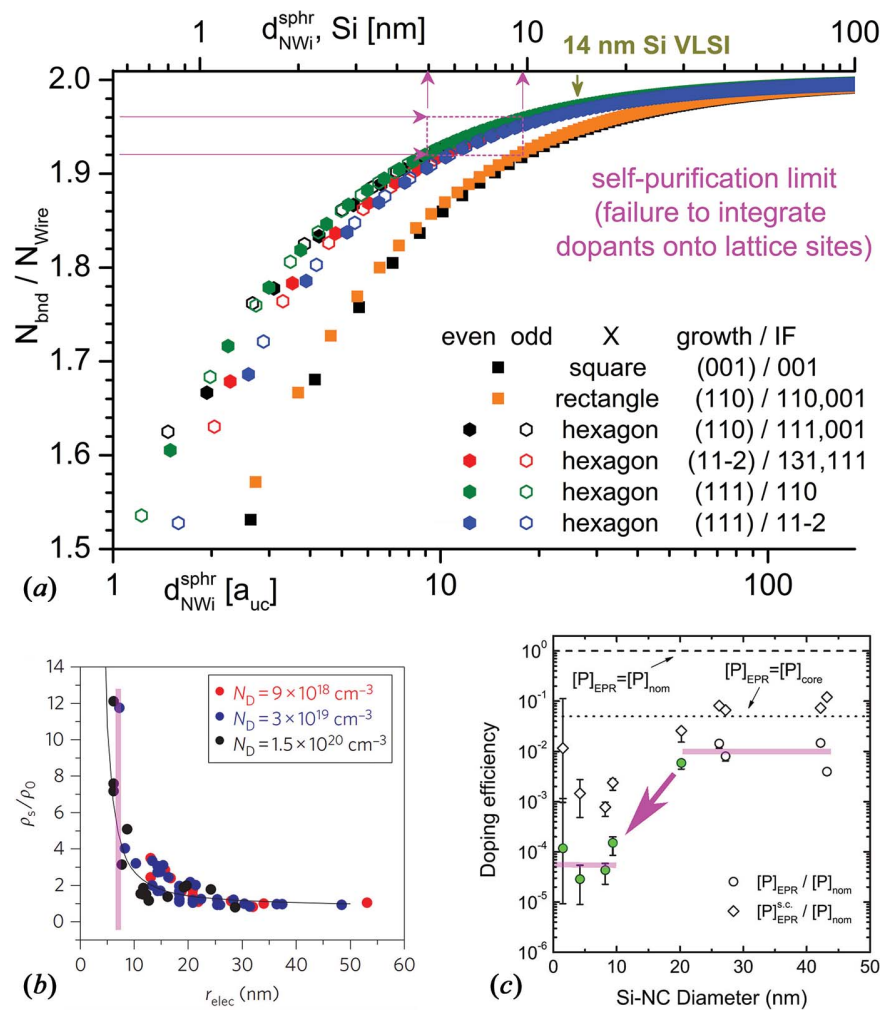

Figure 14

(a) The ratio of internal bonds per NWire atom $N_{\text {bnd }} / N_{\text {Wire }}$ as a function of cross-sectional area expressed by the spherical NWire diameter $d_{\text {Wire }}^{\text {circ }}$ in units of $a_{\mathrm{UC}}$ and absolute values for Si (upper $x$ axis). Magenta labels show the area where self-purification sets in for bottom-up (grown) $\mathrm{Si}$ NWires. (b) The relative increase in Si NWire resistivity over the bulk value as a function of NWire radius (Björk et al., 2009); reproduced with kind permission of Springer Nature, copyright 2009. The magenta strip shows the hard limit where dopant failure occurs. (c) Donors with unpaired electrons within Si NCs (Stegner et al., 2009); reproduced with kind permission of American Physics Society, copyright 2009. Round symbols refer to donors which do not getter Si dangling bonds and can potentially donate an electron at room temperature. Green symbols show regions of interest and magenta lines show the doping probability dropping by a factor of $c a 400$. 
$N_{\mathrm{D}}=1.5 \times 10^{20} \mathrm{~cm}^{-3}$ which present the semiconductor-tosemi-metal limit for Si (Pearson \& Bardeen, 1949), substitutional doping fails for NWire diameters in the range of 7 to $10 \mathrm{~nm}$ (Björk et al., 2009) [Fig. 14(b)]. At this diameter, we have a ratio of $N_{\text {bnd }} / N_{\text {Wire }}=1.94 \pm 0.01$ for Si NWires with hexagonal cross section (Schmid et al., 2008) depending on the interface orientation and growth axis. The values are significantly lower for square and rectangular cross sections. For $\mathrm{Si}$ $\mathrm{NCs}$, the probabilities of built-in dopants dropped 400-fold when decreasing the NC diameters from 20 to $10 \mathrm{~nm}$ (Stegner et al., 2009) [Fig. 14(c)]. For this size range, a ratio of NC internal bonds per NC atom of $N_{\mathrm{bnd}} / N_{\mathrm{NC}}=1.94 \pm 0.02$ was found, where the same analytical description presented here was applied to zb- and diamond-structured NCs (König, 2016). Using independent experimental results from two different nano-Si systems (NWires and NCs), our analytical description shows that $N_{\mathrm{bnd}} / N_{\mathrm{NC}}=1.94 \pm 0.02$ is a general limit below which self-purification prevents effective substitutional doping.

The ratio $N_{\mathrm{IF}}[i] / N_{\text {Wire }}[i]$ yields the number of interface bonds per NWire atom (Fig. 15). This key parameter quantifies electronic phenomena occurring across NWire interfaces. It follows the opposite trend to that discussed for $N_{\text {bnd }}[i] /$ $N_{\text {Wire }}[i]$ : any bond not available for connecting NWire atoms occurs at an interface. NWire cross sections with a high aspect ratio have a higher value of $N_{\mathrm{IF}} / N_{\mathrm{Wire}}$, which results in bigger $d_{\text {Wire }}$ values up to which the embedding dielectric dominates the electronic and optical NWire properties. This finding is important for $\{001\}$-terminated $\mathrm{Si}$ NWires with a square cross section which can be morphed to a high aspect ratio as mentioned at the end of Section 7. Such $\{001\}$-terminated cross sections are encountered in fin-FETs and can be used to exploit nanoscopic phenomena. As an example, the electronic impact of ultrathin $\mathrm{SiO}_{2}$ and $\mathrm{Si}_{3} \mathrm{~N}_{4}$ coatings on $\mathrm{Si}$ NWires with thicknesses as shown in Fig. 13 could replace conventional

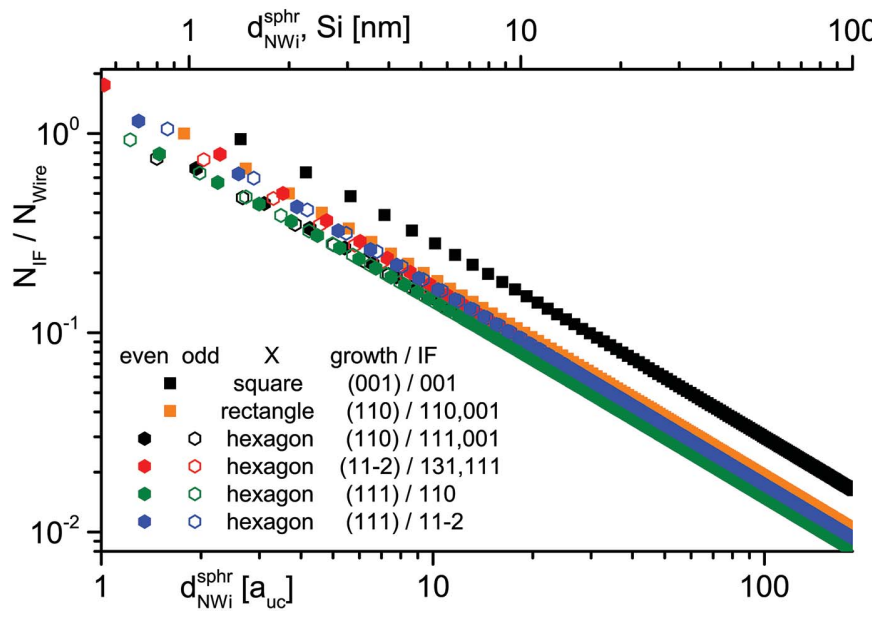

Figure 15

The ratio of interface bonds per NWire atom $N_{\mathrm{IF}} / N_{\mathrm{Wire}}$ as a function of cross-sectional area expressed as the spherical NWire diameter $d_{\text {Wire }}^{\text {circ }}$ in units of $a_{\mathrm{UC}}$ and absolute values for Si (upper $x$ axis) shown for all the NWires of Section 3. doping while maintaining CMOS compatibility for VLSI devices (König et al., 2014, 2018).

The quantity $N_{\mathrm{IF}} / N_{\text {bnd }}$ serves as a gauge for the stress balance between NWires and an embedding matrix or coating interacting via interface bonds, such as GaAs NWires grown with an $\mathrm{Al}_{0.5} \mathrm{In}_{0.5} \mathrm{P}$ shell (Sköld et al., 2006; see Section 4.1). The evolution of $N_{\mathrm{IF}} / N_{\mathrm{bnd}}$ is shown for all NWire cross sections as a function of diameter in Fig. 16. There may exist a minimum of $N_{\mathrm{IF}} / N_{\text {bnd }}$ below which the NWire structure is unstable and may switch to a different cross section or even symmetry group, like the intermittent zb-Würtzite phases of InAs NWires (Park et al., 2015). It is therefore likely that there are certain limits of minimum NWire diameters per crosssection type below which a phase change occurs. This phenomenon was observed for $\mathrm{Si} \mathrm{NCs}$ in $\mathrm{SiO}_{2}$ which have a strong preference for an octahedral shape with $\{111\}$ interfaces for NC sizes of $\leq 3 \mathrm{~nm}$ (Godefroo et al., 2008; König, 2016). Such limits are a function of $N_{\mathrm{IF}} / N_{\text {bnd }}$, the Young's moduli of the semiconductor and its embedding environment, and can be derived for any NWire with appropriate experimental input.

\section{Outlook}

Work is in progress for the NWire cross sections shown here to develop lateral number series which allow asymmetric crosssection morphing to any rectangular aspect ratio for square and rectangular NWires, and from low-aspect hexagonal up to a full rhombic cross section for hexagonal NWires. As an example, square NWire cross sections with an exclusive $\{001\}$ interface could be morphed to large aspect rectangles which describe Si fin-FETs in $14 \mathrm{~nm}$ VLSI technology. Morphed cross sections possess a ratio of interface-specific bonds changing with the shape of the cross section. As an example,

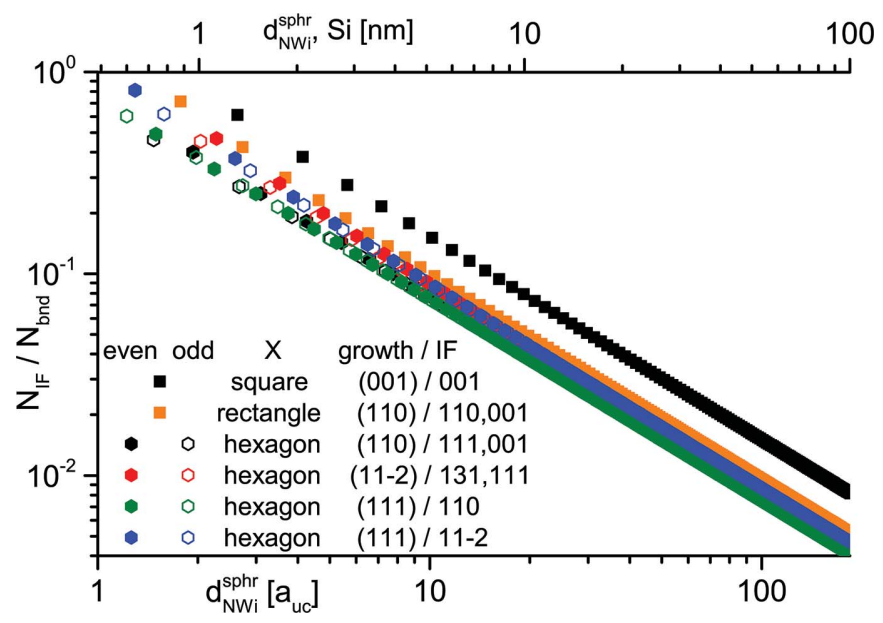

Figure 16

The ratio of interface to internal NWire bonds $N_{\mathrm{IF}} / N_{\text {bnd }}$ as a function of cross-sectional area expressed as the spherical NWire diameter $d_{\text {Wire }}^{\text {circ }}$ in units of $a_{\mathrm{UC}}$ and absolute values for Si (upper $x$ axis) shown for all the NWires of Section 3. 
the ratio of $\{001\}$ to $\{111\}$ interface bonds can be exploited for the interface-specific Si dangling-bond (DB) defects labelled $\mathrm{P}_{\mathrm{b} 0}$ and $\mathrm{P}_{\mathrm{b} 1}$, thereby enabling non-destructive shape detection of NWire cross sections. Such characterization has already been applied to Si NCs (Stesmans et al., 2008) using EPR and recently underpinned by crystallographic analysis (König, 2016).

\section{Conclusions}

We have deduced analytical number series for $\mathrm{zb}$ - and diamond-structured NWires as a function of diameter, shape and interface faceting for the following NWire cross sections: square, $\{001\}$ growth axis and interfaces; rectangular, $\{110\}$ growth axis and $\{110\}$ plus $\{001\}$ interfaces; hexagonal, $\{110\}$ growth axis and $\{001\}$ plus $\{111\}$ interfaces; hexagonal, $\{11 \overline{2}\}$ growth axis and $\{111\}$ plus $\{1 \overline{3} 1\}$ interfaces; hexagonal, $\{111\}$ growth axis and $\{110\}$ interfaces; hexagonal, $\{111\}$ growth axis and $\{11 \overline{2}\}$ interfaces. All hexagonal cross sections are presented in an even and an odd scheme to facilitate matching to the different symmetry centres encountered experimentally. The calculated parameters are the number of NWire atoms $N_{\text {Wire }}[i]$, the number of bonds between such atoms $N_{\text {bnd }}[i]$, the number of NC Wire interface bonds $N_{\mathrm{IF}}[i]$, the interface lengths $d_{\mathrm{IF}}[i]$, the cross-section widths $w[i]$, the heights $h[i]$ and the total cross-section areas $A[i]$. All expressions are linked to the NWire spherical diameters $d_{\text {Wire }}[i]$ to enable direct parameter comparison between different morphologies.

Use of the analytical description has been demonstrated on III-V core-shell and Si NWire STEM/TEM images. The available atomic resolution of the latter allowed exact parameter description down to the atom/bond level, which opens a new avenue to interpret any experimental spectroscopic data of zb- and diamond-structured NWire cross sections.

The ratio $N_{\text {bnd }} / N_{\text {Wire }}$ is useful to gauge the internal stress of NWires, which is key to evaluating self-purification and dopant segregation as encountered in impurity doping, and the general stress response of NWires to an external force. Both $N_{\text {IF }} / N_{\text {bnd }}$ and $N_{\text {bnd }} / N_{\text {Wire }}$ can be applied to optical spectroscopy methods such as FT-IR, Raman, photoluminescence or electroluminescence to interpret and deconvolute spectra into NWire-immanent (internal) and matrix/shell (external) components. The ratio $N_{\mathrm{IF}} / N_{\text {Wire }}$ describes the electronic interaction of NWires with the embedding matrix or ligands to gauge the impact of interface dipoles or interface charge transfer onto the NC electronic structure.

The analytical description of zb- and diamond-structured NWire cross sections could provide major advancement in experimental data interpretation and understanding of III-V, II-VI and group-IV based NWires. The number series allow for a deconvolution of experimental data into environmentexerted, interface-related and NC-internal phenomena. The predictive power of our method could render it an essential tool to predict NWire cross sections and to tune process conditions for tailoring NWires towards desired shapes and interface properties.

\section{APPENDIX $A$}

Derivation of number series, demonstrated for NWires growing along the [001] direction with a square cross section and four $\{001\}$ interfaces (Section 3.1)

This cross section is rather simple in terms of the $\mathrm{zb} /$ diamond structure UC lengths $a_{\mathrm{UC}}$ (Fig. 1) and therefore lends itself to a demonstration of the mathematical tools by which the number series are obtained.

From the side view of the NWire slabs in Fig. 2 we see that $d_{\text {slab }}=1 a_{\mathrm{UC}}$. The smallest NWire cross section (run index $i=1$ ) is shown in Fig. 2(a). It has a side length of the $\{001\}$ interfaces of $d_{001-\mathrm{IF}}^{001-\square}[i=1]=7 / 4 a_{\mathrm{UC}}$. The successive cross section $(i=2)$ has $d_{001-\mathrm{IF}}^{001-\mathrm{I}}[i=2]=11 / 4 a_{\mathrm{UC}}$, then $d_{001-\mathrm{IF}}^{001-\square}[i=3]=15 / 4 a_{\mathrm{UC}}$, or generally

$$
d_{001-\mathrm{IF}}^{001-\mathrm{F}}[i]=a_{\mathrm{UC}}\left(i+\frac{3}{4}\right)
$$

which is identical to equation (5) in Section 3.1. Observe that the side length of the cross section in terms of atomic monolayers (MLs) is $1 / 4 a_{\mathrm{UC}}$ bigger, since the two half-bonds at the interface do not alter the number of atomic MLs. Therefore, the side length of the interfaces in terms of atomic MLs is $i \times a_{\mathrm{UC}}$. This difference of $1 / 4 a_{\mathrm{UC}}$ can be used to countercheck the results on $N_{\text {Wire }}^{001-\square}[i]$ below. Since the cross-section area is $A^{001-\square[i]}=\left(d_{001-\mathrm{IF}}^{001-\mathrm{D}}[i]\right)^{2}$, we arrive directly at equation (6).

To calculate $N_{\text {Wire }}^{001-}[i]$, we use the second-order differential scheme shown below. The values of $N_{\text {Wire }}^{001-}[i]$ are straightforward to count in Fig. 2 using the count per column as outlined in Table 2 - see Table 7.

We see from the differential scheme that the antiderivative of $\mathrm{d}^{2} N_{\text {Wire }}^{001-}[i] / \mathrm{d} i^{2}$ is

$$
\mathrm{d} N_{\text {Wire }}^{001-\square}[i] / \mathrm{d} i=16 i+24,
$$

whereby the number 24 is a constant offset by analogy with an integration constant. The quantity $\mathrm{d} N_{\text {Wire }}^{001-}[i] / \mathrm{d} i$ presents the increment for the transition $N_{\text {Wire }}^{001-\square}[i-1] \rightarrow N_{\text {Wire }}^{001-\square}[i]$, yielding the recursive form of $N_{\text {Wire }}^{001-}[i]$ as

$$
N_{\text {Wire }}^{001-\square}[i]=N_{\text {Wire }}^{001-\square}[i-1]+16(i-1)+24 \text {. }
$$

The recursive nature of $N_{\text {Wire }}^{001-\square}[i]$ means that we add up $\mathrm{d} N_{\text {Wire }}^{001-\square}[k=1,2 \ldots(i-1)] / \mathrm{d} i$ until $i$ reaches the desired value which can be assigned to a certain experimental interface length. Adding up a run index $i$ powered by an exponent $n$ up to $k=i-1$ can be expressed as

$$
1^{n}+2^{n}+3^{n}+\ldots+(i-1)^{n}=\sum_{k=1}^{i-1} k^{n} .
$$

Equation (83) is instrumental in the transition from the recursive to the explicit form of all number series presented in our work. For equation (82) it yields

$$
N_{\text {Wire }}^{001-\square}[i]=16 \sum_{k=1}^{i-1} k^{1}+24 \sum_{k=1}^{i-1} k^{0}=16 \frac{(i-1) i}{2}+24(i-1),
$$


Table 7

Second-order differential scheme for $N_{\text {Wire }}^{001-\square}[i]$.

\begin{tabular}{llll}
\hline$i$ & $N_{\text {Wire }}^{001-\mathrm{\square}}[i]$ & $\mathrm{d} N_{\text {Wire }}^{001-\mathrm{\square}}[i] / \mathrm{d} i$ & $\mathrm{~d}^{2} N_{\text {Wire }}^{001-\mathrm{a}}[i] / \mathrm{d} i^{2}$ \\
\hline 1 & $4 \times 8=32$ & & \\
2 & $6 \times 12=72$ & 40 & \\
3 & $8 \times 16=128$ & 56 & 16 \\
4 & $10 \times 20=200$ & 72 & 16 \\
5 & $12 \times 24=288$ & 88 & 16 \\
6 & $14 \times 28=392$ & 104 & 16 \\
$\cdots$ & $\cdots$ & $\cdots$ & $\cdots$ \\
\hline
\end{tabular}

Table 8

Comparison of counted values of $N_{\mathrm{Wire}}^{001-\mathrm{D}}[i]$ and values derived from equation (84) to show the initial value problem which is analogous to an integration constant.

\begin{tabular}{llll}
\hline$i$ & $\begin{array}{l}N_{\text {Wire }}^{001-}[i] \\
\text { counted }\end{array}$ & $\begin{array}{l}N_{\text {Wire }}^{001-\square}[i] \text { as in } \\
\text { equation }(84)\end{array}$ & $\Delta N_{\text {Wire }}^{001-\square}$ \\
\hline 1 & 32 & 0 & 32 \\
2 & 72 & 40 & 32 \\
3 & 128 & 96 & 32 \\
4 & 200 & 168 & 32 \\
5 & 288 & 256 & 32 \\
6 & 392 & 360 & 32 \\
$\cdots$ & $\cdots$ & $\cdots$ & $\cdots$ \\
\hline
\end{tabular}

where we have used the identity (Zeidler et al., 2004)

$$
\sum_{k=1}^{i} k^{1}=\frac{i(i+1)}{2} .
$$

By analogy with integration yielding an integration constant, an antiderivative possesses a constant offset. This initial-value problem can be solved by comparing the results for each $i$ from equation (84) with the $N_{\mathrm{Wire}}^{001-\square}[i]$ values from the differential scheme above, see Table 8 .

The final explicit form of $N_{\text {Wire }}^{001-}[i]$ is given by adding $\Delta N_{\text {Wire }}^{001-\square}$ to it, viz.

$$
\begin{aligned}
N_{\text {Wire }}^{001-\mathrm{a}}[i] & =16 \frac{(i-1) i}{2}+24(i-1)+32 \\
& =8\left(i^{2}+2 i+1\right) \\
& =8(i+1)^{2},
\end{aligned}
$$

which is identical to equation (2) in Section 3.1.

Another method of calculating $N_{\text {Wire }}^{001-}[i]$ is given by the use of the side length of the square cross section in terms of atomic MLs, which is $d_{001-\mathrm{IF}}^{001-[}[i]=a_{\mathrm{UC}}(i+1)$ (see above for the difference in the actual interface length), and $d_{\text {slab }}=1 a_{\mathrm{UC}}$, with the unit-cell volume of $a_{\mathrm{UC}}^{3}$ containing eight atoms as the smallest increment. This method can be used to counter-check equation (86); see Table 9.

As expected, we arrive at the same result. We point out, though, that for more complex NWire cross sections the latter method becomes very cumbersome.

For $N_{\text {bnd }}^{001-}[i]$, we can repeat the above procedure with different boundary conditions. For internal bonds of the NWire slab, we obtain the differential scheme shown in Table 10.
Table 9

Alternative derivation of $N_{\text {Wire }}^{001-}[i]$ using the side length of the cross section $d_{001-\mathrm{IF}}^{001-}[i]$ with the slab thickness of the cross section of $1 a_{\mathrm{UC}}$ to

\begin{tabular}{|c|c|c|c|}
\hline$i$ & $\begin{array}{l}d_{001-\mathrm{IF}}^{001}[i] \\
\text { for atomic } \\
\operatorname{MLs}\left(a_{\mathrm{UC}}\right)\end{array}$ & $\begin{array}{l}N_{\text {Wire }}^{001-}[i] \\
\text { as in equation } \\
(86) \text { (atoms) }\end{array}$ & $\begin{array}{l}N_{\mathrm{Wire}}^{001-\mathrm{D}}[i] \text { per }\left(d_{001-\mathrm{IF}}^{001-\mathrm{I}}[i]\right)^{2} \times d_{\text {slab }} \\
\left(a_{\mathrm{UC}}\right)^{3}(\text { atoms })\end{array}$ \\
\hline 1 & 2 & 32 & $4 ; 32$ \\
\hline 2 & 3 & 72 & $9 ; 72$ \\
\hline 3 & 4 & 128 & $16 ; 128$ \\
\hline 4 & 5 & 200 & $25 ; 200$ \\
\hline 5 & 6 & 288 & $36 ; 288$ \\
\hline 6 & 7 & 392 & $49 ; 392$ \\
\hline$\cdots$ & $\ldots$ & $\cdots$ & $\cdots$ \\
\hline
\end{tabular}
arrive at the slab volume in units of $\left(a_{\mathrm{UC}}\right)^{3}$ and thus $N_{\text {Wire }}^{001-}[i]$ due to the volume of $\left(a_{\mathrm{UC}}\right)^{3}$ containing 8 or $4+4$ atoms for diamond and $\mathrm{zb}$ structures, respectively.

Table 10 Second-order differential scheme for $N_{\mathrm{bnd}}^{001-\square}[i]$.

\begin{tabular}{llll}
\hline$i$ & $N_{\text {bnd }}^{001-\square}[i]$ & $\mathrm{d} N_{\text {bnd }}^{001-\mathrm{D}}[i] / \mathrm{d} i$ & $\mathrm{~d}^{2} N_{\mathrm{bnd}}^{001-\mathrm{a}}[i] / \mathrm{d} i^{2}$ \\
\hline 1 & $7 \times 7=49$ & & \\
2 & $11 \times 11=121$ & 72 & \\
3 & $15 \times 15=225$ & 104 & 32 \\
4 & $19 \times 19=361$ & 136 & 32 \\
5 & $23 \times 23=529$ & 168 & 32 \\
6 & $27 \times 27=729$ & 200 & 32 \\
$\cdots$ & $\cdots$ & $\cdots$ & $\cdots$ \\
\hline
\end{tabular}

Table 11

First-order differential scheme for $N_{\mathrm{IF}}^{001-\square}[i]$.

\begin{tabular}{lll}
\hline$i$ & $N_{\mathrm{IF}}^{001-\square}[i]$ & $\mathrm{d} N_{\mathrm{IF}}^{001-\square}[i] / \mathrm{d} i$ \\
\hline 1 & $4(3 \times 2)+2 \times 3=30$ & \\
2 & $4(5 \times 2)+2 \times 3=46$ & 16 \\
3 & $4(7 \times 2)+2 \times 3=62$ & 16 \\
4 & $4(9 \times 2)+2 \times 3=78$ & 16 \\
5 & $4(11 \times 2)+2 \times 3=94$ & 16 \\
6 & $4(13 \times 2)+2 \times 3=110$ & 16 \\
$\cdots$ & $\cdots$ & $\cdots$ \\
\hline
\end{tabular}

From column 2 of Table 10 we can see that the square product scales with $i$ since $N_{\mathrm{bnd}}^{001-\mathrm{q}}[1]=(1 \times 4+3)^{2}=7^{2}$, $N_{\text {bnd }}^{001-\square}[2]=(2 \times 4+3)^{2}=11^{2}, N_{\text {bnd }}^{\text {ond }}$ [ $[3]=(3 \times 4+3)^{2}=15^{2}$, etc .

This shortcut leads us straight to the solution,

$$
N_{\mathrm{bnd}}^{001-\mathrm{a}}[i]=(4 i+3)^{2},
$$

which is equation (3) in Section 3.1. The interested reader can run the diffential scheme outlined above on $N_{\text {bnd }}^{001-}[i]$ to become familiar with the algorithm.

For $N_{\mathrm{IF}}^{001-}[i]$, we use the colour-coded interface termination: red atoms have one interface bond, blue atoms have two interface bonds and green atoms have three interface bonds; see also Section 2. The respective number of interface bonds per run index $i$ as counted in Fig. 2 is given in Table 11.

From $\mathrm{d} N_{\mathrm{IF}}^{001-}-[i] / \mathrm{d} i$ we get the recursive form

$$
N_{\mathrm{IF}}^{001-\mathrm{q}}[i]=N_{\mathrm{IF}}^{001-\mathrm{q}}[i-1]+16 .
$$

The constant increment accounts for the linear nature of the IF length with run index $i$ (versus the quadratic dependence of $N_{\text {Wire }}^{001-\mathrm{D}}[i]$ and $N_{\mathrm{bnd}}^{001-\mathrm{\square}}[i]$ as variables related to cross-section 
area). With equation (88), we get $N_{\mathrm{IF}}^{001-}{ }^{-}[1]=16, N_{\mathrm{IF}}^{001-\mathrm{\square}}[2]=$ $32, N_{\mathrm{IF}}^{001-}[3]=48$ etc., which differ from the counted values in the differential scheme above by an integration constant of 14 . Using equation (83), we get $N_{\mathrm{IF}}^{001-}[i]$ in its explicit form

$$
N_{\mathrm{IF}}^{001-\square}[i]=16 i+14=2(8 i+7) \text {, }
$$

which is identical to equation (5) in Section 3.1.

\section{Funding information}

D. König acknowledges funding from the 2015 University of New South Wales Blue Sky Research Grant (award No. PS37884), the 2012, 2014 and 2016 DAAD-Go8 Joint Research Cooperation Schemes and the 2018 Theodore-vonKàrmàn Fellowship of RWTH Aachen University, Germany.

\section{References}

Anastassakis, E., Pinczuk, A., Burstein, E., Pollak, F. H. \& Cardona, M. (1970). Solid State Commun. 8, 133-138.

Björk, M. T., Schmid, H., Knoch, J., Riel, H. \& Riess, W. (2009). Nat. Nanotechnol. 4, 103-107.

Böer, K. W. (1990). Survey of Semiconductor Physics, Vol. 1. New York: Van Nostrand Reinhold.

Boyd, I. W. \& Wilson, J. I. B. (1982). J. Appl. Phys. 53, 4166-4172.

Boyd, I. W. \& Wilson, J. I. B. (1987). J. Appl. Phys. 62, 3195-3200.

Chan, T.-L., Tiago, M. L., Kaxiras, E. \& Chelikowsky, J. R. (2008). Nano Lett. 8, 596-600.

Dalpian, G. M. \& Chelikowsky, J. R. (2006). Phys. Rev. Lett. 96, 226802.

Dalpian, G. M. \& Chelikowsky, J. R. (2008). Phys. Rev. Lett. 100, 179703.

Eaglesham, D. J., White, A. E., Feldman, L. C., Moriya, N. \& Jacobson, D. C. (1993). Phys. Rev. Lett. 70, 1643-1646.

Elliot, S. R. (1998). The Physics and Chemistry of Solids. Chichester: Wiley.

Gnaser, H., Gutsch, S., Wahl, M., Schiller, R., Kopnarski, M., Hiller, D. \& Zacharias, M. (2014). J. Appl. Phys. 115, 034304.

Godefroo, S., Hayne, M., Jivanescu, M., Stesmans, A., Zacharias, M., Lebedev, O. I., Van Tendeloo, G. \& Moshchalkov, V. V. (2008). Nat. Nanotechnol. 3, 174-178.

Hesketh, P. J. (1993). J. Electrochem. Soc. 140, 1080-1085.

Hiller, D., López-Vidrier, J., Gutsch, S., Zacharias, M., Nomoto, K. \& König, D. (2017a). Sci. Rep. 7, 863.

Hiller, D., López-Vidrier, J., Gutsch, S., Zacharias, M., Wahl, M., Bock, W., Brodyanski, A., Kopnarski, M., Nomoto, K., Valenta, J. \& König, D. (2017b). Sci. Rep. 7, 8337.
Jaccodine, R. J. \& Schlegel, W. A. (1966). J. Appl. Phys. 37, 24292434.

Joyce, H. J., Gao, Q., Hoe Tan, H., Jagadish, C., Kim, Y., Zou, J., Smith, L. M., Jackson, H. E., Yarrison-Rice, J. M., Parkinson, P. \& Johnston, M. B. (2011). Prog. Quantum Electron. 35, 23-75.

König, D. (2016). AIP Adv. 6, 085306.

König, D., Gutsch, S., Gnaser, H., Wahl, M., Kopnarski, M., Göttlicher, J., Steininger, R., Zacharias, M. \& Hiller, D. (2015). Sci. Rep. 5, 9702.

König, D., Hiller, D., Gutsch, S. \& Zacharias, M. (2014). Adv. Mater. Interfaces, 1, 1400359.

König, D., Hiller, D., Wilck, N., Berghoff, B., Müller, M., Thakur, S., Di Santo, G., Petaccia, L., Mayer, J., Smith, S. \& Knoch, J. (2018). Beilstein J. Nanotechnol. 9, 2255-2264.

König, D., Rennau, M. \& Henker, M. (2007). Solid-State Electron. 51, 650-654.

Nakashima, S., Oima, S., Mitsuishi, A., Nishimura, T., Fukumoto, T. \& Akasaka, Y. (1981). Solid State Commun. 40, 765-768.

Ossicini, S., Degoli, E., Iori, F., Luppi, E., Magri, R., Cantele, G., Trani, F. \& Ninno, D. (2005). Appl. Phys. Lett. 87, 173120.

Park, D. W., Jeon, S. G., Lee, C.-R., Lee, S. J., Song, J. Y., Kim, J. O., Noh, S. K., Leem, J.-Y. \& Kim, J. S. (2015). Sci. Rep. 5, 16652.

Pearson, G. L. \& Bardeen, J. (1949). Phys. Rev. 75, 865-883.

Schmid, H., Björk, M. T., Knoch, J., Riel, H., Riess, W., Rice, P. \& Topuria, T. (2008). J. Appl. Phys. 103, 024304.

Sköld, N., Wagner, J. B., Karlsson, G., Hernán, T., Seifert, W., Pistol, M.-E. \& Samuelson, L. (2006). Nano Lett. 6, 27432747.

Stegner, A. R., Pereira, R. N., Klein, K., Lechner, R., Dietmueller, R., Brandt, M. S., Stutzmann, M. \& Wiggers, H. (2008). Phys. Rev. Lett. 100, 026803.

Stegner, A. R., Pereira, R. N., Lechner, R., Klein, K., Wiggers, H., Stutzmann, M. \& Brandt, M. S. (2009). Phys. Rev. B, 80, 165326.

Stesmans, A., Jivanescu, M., Godefroo, S. \& Zacharias, M. (2008). Appl. Phys. Lett. 93, 023123.

Tomioka, K., Tanaka, T., Hara, S., Hiruma, K. \& Fukui, T. (2011). Quantum Electron. 17, 1112-1129.

Treu, J., Stettner, T., Watzinger, M., Morkötter, S., Döblinger, M., Matich, S., Saller, K., Bichler, M., Abstreiter, G., Finley, J. J., Stangl, J. \& Koblmüller, G. (2015). Nano Lett. 15, 35333540 .

Weber, W. M. \& Mikolajick, T. (2017). Rep. Prog. Phys. 80, 066502.

Yi, K. S., Trivedi, K., Floresca, H. C., Yuk, H., Hu, W. \& Kim, M. J. (2011). Nano Lett. 11, 5465-5470.

Zeidler, E., Hackbusch, W. \& Schwarz, H. R. (2004). Oxford User's Guide to Mathematics. Translated from German by Bruce Hunt. Oxford University Press. 